\title{
Inference Under a Wright-Fisher Model Using an Accurate Beta Approximation
}

\author{
Paula Tataru, ${ }^{1}$ Thomas Bataillon, and Asger Hobolth \\ Bioinformatics Research Centre, Aarhus University, Aarhus C 8000, Denmark
}

\begin{abstract}
The large amount and high quality of genomic data available today enable, in principle, accurate inference of evolutionary histories of observed populations. The Wright-Fisher model is one of the most widely used models for this purpose. It describes the stochastic behavior in time of allele frequencies and the influence of evolutionary pressures, such as mutation and selection. Despite its simple mathematical formulation, exact results for the distribution of allele frequency (DAF) as a function of time are not available in closed analytical form. Existing approximations build on the computationally intensive diffusion limit or rely on matching moments of the DAF. One of the moment-based approximations relies on the beta distribution, which can accurately describe the DAF when the allele frequency is not close to the boundaries ( 0 and 1$)$. Nonetheless, under a Wright-Fisher model, the probability of being on the boundary can be positive, corresponding to the allele being either lost or fixed. Here we introduce the beta with spikes, an extension of the beta approximation that explicitly models the loss and fixation probabilities as two spikes at the boundaries. We show that the addition of spikes greatly improves the quality of the approximation. We additionally illustrate, using both simulated and real data, how the beta with spikes can be used for inference of divergence times between populations with comparable performance to an existing state-of-the-art method.
\end{abstract}

KEYWORDS Wright-Fisher; beta; pure genetic drift; linear evolutionary pressures; divergence times

DVANCES in sequencing technologies have revolutionized the collection of genomic data, increasing both the volume and quality of available sequenced individuals from a large variety of populations and species (Romiguier et al. 2014; Gudbjartsson et al. 2015). These data, which may involve up to millions of single-nucleotide polymorphisms (SNPs), contain information about the evolutionary history of the observed populations. There has been a great focus in the recent years on inferring such histories, and to this end, one of the most widely used models is the Wright-Fisher model (Gautier et al. 2010; Sirén et al. 2011; Malaspinas et al. 2012; Pickrell and Pritchard 2012; Gautier and Vitalis 2013; Steinrücken et al. 2014; Terhorst et al. 2015).

The Wright-Fisher model characterizes the evolution of a randomly mating population of finite size in discrete nonoverlapping generations. The model describes the sto-

Copyright @ 2015 by the Genetics Society of America

doi: 10.1534/genetics.115.179606

Manuscript received June 19, 2015; accepted for publication August 22, 2015; published Early Online August 26, 2015.

Supporting information is available online at www.genetics.org/lookup/suppl/ doi:10.1534/genetics.115.179606/-/DC1

${ }^{1}$ Corresponding author: Bioinformatics Research Centre, Aarhus University, C. F.

Møllers Allé 8, Aarhus C 8000, Denmark. E-mail: paula@cs.au.dk chastic behavior in time of the number of copies (frequency) of alleles at a locus. The frequency is influenced by a series of factors, such as random genetic drift, mutations, migrations, selection, and changes in population size. When inferring the evolutionary history of a population, the effects of the different factors have to be untangled. Mutation, migration, and selection affect the allele frequency in a deterministic manner (Ewens 2004). We collectively refer to these as evolutionary pressures. The frequency also varies from one generation to the next as a result of random sampling of a finite-sized population (random genetic drift). Mutations and migrations result in linear changes of the sampling probability, while selection is a nonlinear pressure (Kimura 1964; Crow and Kimura 1970) and therefore is more difficult to study analytically.

A crucial step for carrying out statistical inference in the Wright-Fisher model is determination of the distribution of allele frequency (DAF) as a function of time, conditional on an initial frequency. Even though the Wright-Fisher model has a very simple mathematical formulation, no tractable analytical form exists for the DAF (Ewens 2004). Therefore, various approximations have been developed, ranging from purely analytical to purely numerical. They generally either 
build on the diffusion limit of the Wright-Fisher model or rely on matching moments of the true DAF. Both types of approximations have been used successfully for inference of populations divergence times (Sirén et al. 2011; Gautier and Vitalis 2013), populations admixture (Pickrell and Pritchard 2012), SNPs under selection (Gautier et al. 2010), and selection coefficients from time-serial data (Malaspinas et al. 2012; Steinrücken et al. 2014; Terhorst et al. 2015).

Wright (1945) was the first to use the diffusion approximation to determine the stationary DAF. Kimura (1955) solved the diffusion limit and found the time-dependent distribution for pure genetic drift, and Crow and Kimura (1956) extended the solution to include linear evolutionary pressures. However, these approaches contain infinite sums, making their use cumbersome in practice. After decades dominated by inference based on the dual coalescent process (Rosenberg and Nordborg 2002; Hoban et al. 2012), diffusion has recently received increasing attention, and researchers have started to investigate other ways to solve analytically or approximate the diffusion equation (McKane and Waxman 2007; Waxman 2011; Malaspinas et al. 2012; Song and Steinrücken 2012; Zhao et al. 2013; Steinrücken et al. 2013, 2014).

Moment-based approximations are less ambitious in that they aim at fitting mathematically convenient distributions by equating the first moments of the true DAF. Such approximations typically use either the normal distribution (Nicholson et al. 2002; Coop et al. 2010; Gautier et al. 2010; Pickrell and Pritchard 2012; Terhorst et al. 2015) or the beta distribution (Balding and Nichols 1995, 1997; Sirén et al. 2011; Sirén 2012). The rationale behind the use of these distributions is twofold. First, they are motivated by the diffusion limit: the normal distribution is the resulting DAF when drift is small (Nicholson et al. 2002), while the beta distribution is the stationary DAF under linear evolutionary pressures (Wright 1945; Crow and Kimura 1956). Second, they are entirely determined by their mean and variance. One major difference between the two is their support. Because the normal distribution is defined over the whole real line, it needs to be truncated to $[0,1]$ (Nicholson et al. 2002; Coop et al. 2010; Gautier et al. 2010). The truncated normal distribution has two atoms at 0 and 1 (corresponding to the allele being lost or fixed) containing the densities in the intervals $(-\infty, 0]$ and $[1, \infty)$, respectively. However, the truncation procedure leads to a variance that no longer matches the variance of the true DAF (Gautier and Vitalis 2013). Alternatively, the full distribution can be applied for intermediary frequencies only, when the probabilities of lying outside the 0 and 1 boundaries are small and therefore can be ignored (Pickrell and Pritchard 2012; Terhorst et al. 2015). Unlike the normal distribution, the beta distribution has the interval $[0,1]$ as support, but because of its continuous nature, the probabilities at the boundaries will always be 0 . Under a WrightFisher model, the loss and fixation events have a positive probability. The beta distribution provides a good fit for in- termediary frequencies but fails at capturing the nonzero boundary probabilities, as illustrated for pure genetic drift in Figure 1, A-C. When time is small, most of the probability mass is found close to the initial value $x_{0}$ (Figure 1A). As time becomes larger, the allele frequency drifts away from $x_{0}$, and more and more probability accumulates at the boundaries (Figure 1, B and C).

Here we propose an accurate extension of the beta distribution under linear evolutionary pressures called beta with spikes that explicitly models the probabilities at the boundaries. We show that the addition of spikes greatly improves the fit to the true DAF. We use simulation experiments and published chimpanzee exome data to demonstrate that beta with spikes can be used for inference of population divergence times under pure genetic drift with performance comparable to that of a state-of-the-art diffusion-based method (Gautier and Vitalis 2013) and less computational burden. We additionally discuss how beta with spikes can be used in future development to account for variable population size and selection.

\section{Beta with Spikes Approximation}

Consider a diploid randomly mating population of size $2 \mathrm{~N}$ and a biallelic locus with alleles $A_{1}$ and $A_{2}$. Under a Wright-Fisher model, the count of one of the alleles, $A_{1}$, at the discrete generation $t$ is a random variable $Z_{t} \in\{0,1, \ldots, 2 N\}$. Let $X_{t}=Z_{t} /(2 N)$ be the corresponding allele frequency. The evolution of $Z_{t}$ is shaped by random genetic drift and evolutionary pressures that affect the sampling probability (Ewens 2004). We capture the joint effect of the evolutionary pressures in $g(x)$, a polynomial in the allele frequency $0 \leq x \leq 1$. Conditional on $Z_{t}, Z_{t+1}$ follows a binomial distribution (Ewens 2004)

$$
Z_{t+1} \mid Z_{t}=z_{t} \sim \operatorname{Bin}\left[2 N, g\left(x_{t}\right)\right]
$$

Here we consider only linear evolutionary pressures, such as mutation and migration. Then $g(x)$ takes the form

$$
g(x)=(1-a) x+b
$$

Because $g(x)$ represents the sampling probability in equation (1), we must have $0 \leq g(x) \leq 1$, for all $0 \leq x \leq 1$. From this we find that $a$ and $b$ satisfy $0 \leq b \leq a \leq 1$. The case where $a=1$, for which $g(x)=b$, for all $0 \leq x \leq 1$, has no biological meaning, and we therefore assume that $a \neq 1$.

Under pure genetic drift, $a=b=0$. If mutations happen with probabilities $u$ (from $A_{1}$ to $A_{2}$ ) and $v$ (from $A_{2}$ to $A_{1}$ ), then $a=u+v$ and $b=v$. Migration can be modeled, for example, by assuming that individuals can migrate away from the population under study and that there is an influx of individuals from a large population with constant frequency $x_{c}$. Then, with probabilities $m_{1}$ and $m_{2}$, individuals migrate from and to the population under study, respectively. We have $a=m_{1}$ and $b=m_{2} x_{c}$. Mutation and migration can be modeled jointly, resulting in $a=m_{1}+\left(1-m_{1}\right)(u+v)$ and 

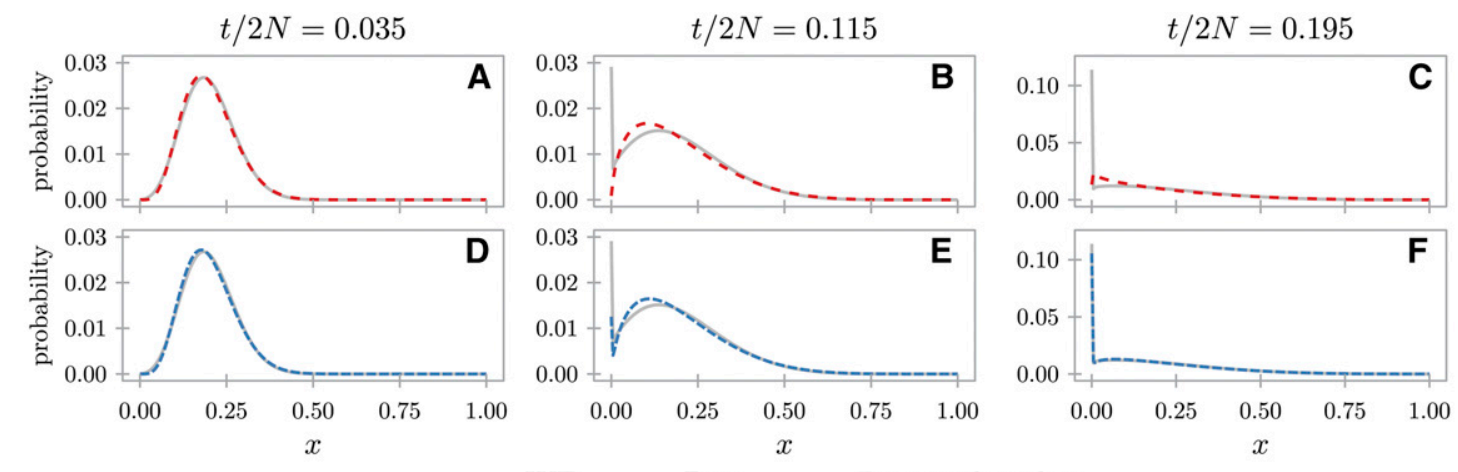

WF - - - Beta ---- Beta with spikes
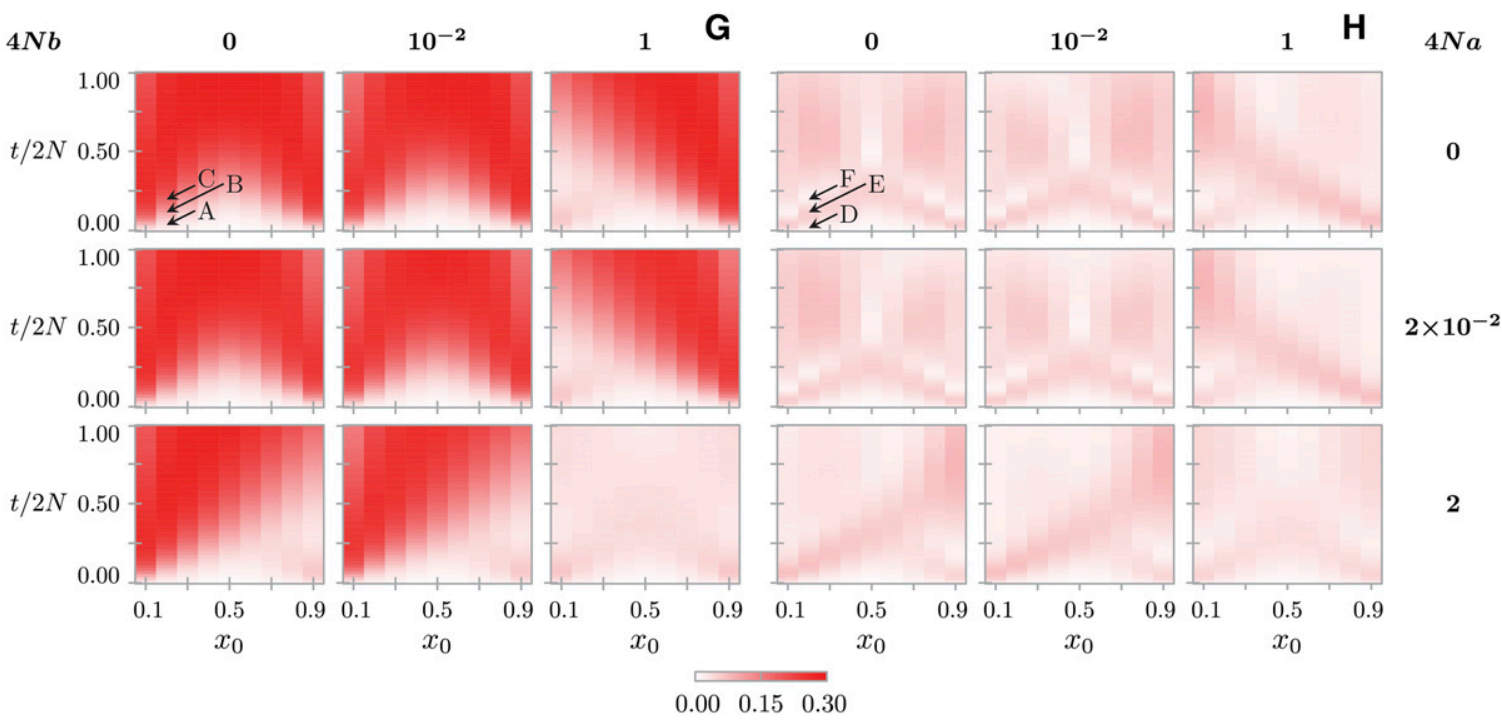

Figure 1 Fit of the beta and beta with spikes approximations for a population of size $2 N=200$. (A-F) The true discrete $D A F$ as given by the Wright-Fisher model under pure genetic drift and the corresponding discretized beta $(A-C)$ and beta with spikes (D-F) approximations. The distributions are conditional on an initial frequency $x_{0}=0.2$ and for different time points $t / 2 N=0.035(A, D), t / 2 N=0.115(B, E)$, and $t / 2 N=0.195(C, F)$, where $t$ is the number of discrete generations that the population has evolved. $(G, H)$ The Hellinger distance between the true DAF and the beta $(\mathrm{G})$ and beta with spikes $(\mathrm{H})$ as a function of $x_{0}$ and $t / 2 N$. Each row and column corresponds to specific values of the scaled parameters $4 \mathrm{Na}$ and $4 \mathrm{Nb}$. The distances corresponding to the distributions from A-F are marked with arrows. The discretization procedure and Hellinger distance are detailed in File S1.

$b=\left(1-m_{1}\right) v+m_{2} x_{c}$. In the following, we treat the general linear case.

We are interested in the DAF $X_{t}$ conditional on $X_{0}=x_{0}$ as a function of the generation $t$

$$
f(x ; t)=\mathbb{P}\left(X_{t}=x \mid X_{0}=x_{0}\right)
$$

For simpler notation, we leave out the explicit condition on $X_{0}=x_{0}$ and implicit condition on population size and evolutionary pressures.

Under the beta approximation, the DAF is (Balding and Nichols 1995)

$$
f_{B}(x ; t)=\frac{x^{\alpha_{t}-1}(1-x)^{\beta_{t}-1}}{\mathrm{~B}\left(\alpha_{t}, \beta_{t}\right)}
$$

where $\mathrm{B}(\alpha, \beta)$ is the beta function. The two shape parameters of the beta distribution are entirely determined by its mean and variance

$$
\begin{aligned}
& \alpha_{t}=\left[\frac{\mathbb{E}\left[X_{t}\right]\left(1-\mathbb{E}\left[X_{t}\right]\right)}{\operatorname{Var}\left(X_{t}\right)}-1\right] \mathbb{E}\left[X_{t}\right] \\
& \beta_{t}=\left[\frac{\mathbb{E}\left[X_{t}\right]\left(1-\mathbb{E}\left[X_{t}\right]\right)}{\operatorname{Var}\left(X_{t}\right)}-1\right]\left(1-\mathbb{E}\left[X_{t}\right]\right)
\end{aligned}
$$

Therefore, in order to fit $f_{B}$ to $f$, we need to calculate $\mathbb{E}\left[X_{t}\right]$ and $\operatorname{Var}\left(X_{t}\right)$. These can be obtained in closed analytical form (see Supporting Information, File S1 for a full derivation). The mean is entirely determined by the initial frequency $x_{0}$ and the parameters $a$ and $b$ of the linear evolutionary pressures, while the variance also depends on the population size. Under pure genetic drift $(a=b=0)$, we have

$$
\begin{aligned}
\mathbb{E}\left[X_{t}\right] & =x_{0} \\
\operatorname{Var}\left(X_{t}\right) & =x_{0}\left(1-x_{0}\right)\left[1-\left(1-\frac{1}{2 N}\right)^{t}\right]
\end{aligned}
$$


When $a \neq 0$, we get

$$
\begin{aligned}
\mathbb{E}\left[X_{t}\right]= & \frac{b}{a}+(1-a)^{t}\left(x_{0}-\frac{b}{a}\right) \\
\operatorname{Var}\left(X_{t}\right)= & \frac{b}{a}\left(1-\frac{b}{a}\right) \frac{1-(1-a)^{2 t}\left(1-\frac{1}{2 N}\right)^{t}}{2 N-(1-a)^{2}(2 N-1)} \\
& +\left(1-\frac{2 b}{a}\right)\left(x_{0}-\frac{b}{a}\right)(1-a)^{t} \frac{1-(1-a)^{t}\left(1-\frac{1}{2 N}\right)^{t}}{2 N-(1-a)(2 N-1)} \\
& -\left(x_{0}-\frac{b}{a}\right)^{2}(1-a)^{2 t}\left[1-\left(1-\frac{1}{2 N}\right)^{t}\right]
\end{aligned}
$$

In the limit of infinite population size, the preceding formulas are equivalent to the mean and variance obtained by Sirén (2012). We note that this equation corrects some typographic errors in Sirén (2012), as confirmed by correspondence with the author (see also File S1).

To account for loss and fixation probabilities, we surround the beta distribution with two spikes

$$
\begin{aligned}
f_{B}^{\star}(x ; t)= & \mathbb{P}\left(X_{t}=0\right) \cdot \delta(x)+\mathbb{P}\left(X_{t}=1\right) \cdot \delta(1-x) \\
& +\mathbb{P}\left(X_{t} \notin\{0,1\}\right) \cdot \frac{x^{\alpha_{t}^{\star}-1}(1-x)^{\beta_{t}^{\star}-1}}{\mathrm{~B}\left(\alpha_{t}^{\star}, \beta_{t}^{\star}\right)}
\end{aligned}
$$

where $\delta(x)$ is the Dirac delta function, and $\mathbb{P}\left(X_{t} \notin\{0,1\}\right)=$ $1-\mathbb{P}\left(X_{t}=0\right)-\mathbb{P}\left(X_{t}=1\right)$. The incorporation of the loss and fixation probabilities into the DAF by means of Dirac delta functions has also been used to obtain a complete solution of the diffusion equation (McKane and Waxman 2007).

To fit $f_{B}^{\star}$ to $f$, we need to determine the mean and variance of $X_{t}$ conditional on polymorphism $\left(X_{t} \notin\{0,1\}\right)$ and the probabilities $\mathbb{P}\left(X_{t}=0\right)$ and $\mathbb{P}\left(X_{t}=1\right)$ of loss and fixation, respectively. Given $\mathbb{E}\left[X_{t}\right], \operatorname{Var}\left(X_{t}\right), \mathbb{P}\left(X_{t}=0\right)$, and $\mathbb{P}\left(X_{t}=1\right)$, the conditional mean and variance can easily be calculated (see File S1). The shape parameters $\alpha_{t}^{\star}$ and $\beta_{t}^{\star}$ are calculated as in equation (5), where the mean and variance are replaced by the conditional mean and variance, respectively. Therefore, we only require a means of calculating the loss and fixation probabilities to fully specify the beta with spikes approximation. We use a recursive approach in which we calculate the probabilities for $X_{t+1}$ by relying on the approximated $f_{B}^{\star}(x ; t)$. We additionally assume that $a$ and $b$ are small to obtain the following approximation for loss and fixation probabilities (see File S1 for a full derivation):

$$
\begin{aligned}
\mathbb{P}\left(X_{t+1}=\right. & 0) \approx \mathbb{P}\left(X_{t}=0\right) \cdot(1-b)^{2 N}+\mathbb{P}\left(X_{t}=1\right) \cdot(a-b)^{2 N} \\
& +\mathbb{P}\left(X_{t} \notin\{0,1\}\right) \cdot(1-a)^{2 N} \frac{\mathrm{B}\left(\alpha_{t}^{\star}, \beta_{t}^{\star}+2 N\right)}{\mathrm{B}\left(\alpha_{t}^{\star}, \beta_{t}^{\star}\right)}
\end{aligned}
$$

$$
\begin{aligned}
\mathbb{P}\left(X_{t+1}=1\right) \approx & \mathbb{P}\left(X_{t}=0\right) \cdot b^{2 N}+\mathbb{P}\left(X_{t}=1\right) \cdot(1-a+b)^{2 N} \\
& +\mathbb{P}\left(X_{t} \notin\{0,1\}\right) \cdot(1-a)^{2 N} \frac{\mathrm{B}\left(\alpha_{t}^{\star}+2 N, \beta_{t}^{\star}\right)}{\mathrm{B}\left(\alpha_{t}^{\star}, \beta_{t}^{\star}\right)}
\end{aligned}
$$

Figure 1, D-F depicts the beta with spikes approximation for the same cases as in Figure 1, A-C. When time is small (Figure $1, \mathrm{~A}$ and $\mathrm{D}$ ), the beta and beta with spikes distributions are equivalent, but as the time becomes larger, the advantage of adding the spikes becomes evident.

To investigate the approximation quality of the beta with spikes, we calculated the Hellinger distance between the true DAF and the beta and beta with spikes for a range of initial frequencies $x_{0}$, times $t$ and parameters $a$ and $b$ (see File S1 for details). The Hellinger distance lies between 0 and 1 , with 0 indicating a perfect match between the two distributions. Figure 1, G and H, shows that the addition of spikes drastically improves the fit of the beta approximation to the true DAF under a Wright-Fisher model. It is apparent from the figure that the beta distribution approximates well the true DAF when it is not close to the boundaries: either the initial frequency is close to 0.5 and the time is not too large or the parameters $a$ or $b$ are large enough to keep the allele frequency away from 0 and 1. The beta with spikes has a more consistent behavior because the corresponding Hellinger distance does not vary as much across the different parameters as it does for the beta distribution.

\section{Inference of Divergence Times}

To further illustrate the advantage of incorporating the spikes, we inferred divergence times between populations using both simulated data and exome sequencing data from three chimpanzee subspecies (Bataillon et al. 2015).

Populations are represented as successive descendants of a single ancestral population. We assume that after each split, the new populations evolve in isolation (no migration) under pure genetic drift. A rooted tree (Figure 2) can be used to describe the joint history of several present populations, located at the leaves, while the common ancestral population is represented as the root. The data $\mathcal{D}=\left\{\left(z_{i j}, n_{i j}\right) \mid 1 \leq i \leq I, 1 \leq j \leq J\right\}$ consist of $I$ independent SNPs for $J$ populations in the present: the (arbitrarily defined) reference $\left(A_{1}\right)$ allele count $z_{i j}$ in a sample of size $n_{i j}\left(0 \leq z_{i j} \leq n_{i j}\right)$ for each locus $1 \leq i \leq I$ and population $1 \leq j \leq J$.

Conditional on the topology (i.e., tree without branch lengths), we inferred the scaled branch lengths by numerically maximizing the likelihood of the data.

\section{Likelihood of the data}

Assuming Hardy-Weinberg equilibrium, the probability of observing $z_{i j}$ alleles in a sample of size $n_{i j}$ given the population allele frequency $x_{i j}$ is given by the binomial distribution 
Figure 2 History of three populations in the present. Ancestral population 5

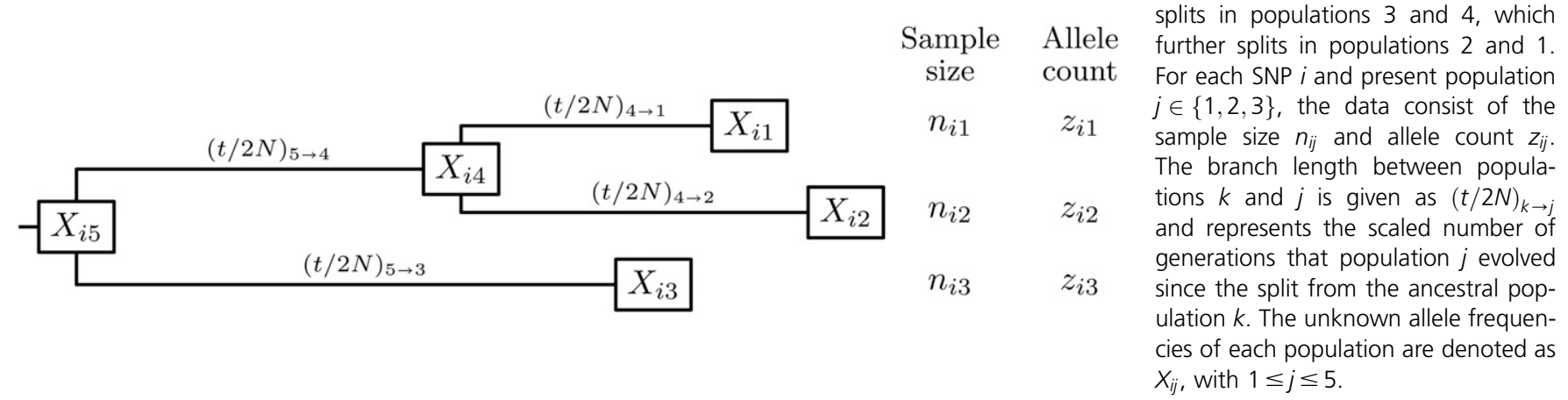

$$
\mathbb{P}\left(z_{i j} \mid n_{i j}, x_{i j}\right)=\left(\begin{array}{c}
n \\
z_{i j}
\end{array}\right) x_{i j}^{z_{i j}}\left(1-x_{i j}\right)^{n_{i j}-z_{i j}}
$$

However, the allele frequencies $x_{i j}$ are unobserved, and the likelihood of the data $\mathcal{D}_{i}$ for SNP $i$ is obtained by integrating over the unknown allele frequencies

$$
\begin{aligned}
L\left(\mathcal{D}_{i} ; \Theta, \pi\right)= & \int_{0}^{1} \ldots \int_{0}^{1} f\left(X_{i 1}, X_{i 2}, \ldots, X_{i J} \mid \Theta, \pi\right) \\
& \cdot \prod_{j=1}^{J} \mathbb{P}\left(z_{i j} \mid n_{i j}, X_{i j}\right) \mathrm{d} X_{i 1} \cdots \mathrm{d} X_{i J}
\end{aligned}
$$

where $f\left(X_{i 1}, X_{i 2}, \ldots, X_{i J} \mid \Theta, \pi\right)$ is the joint distribution of the $X_{i j}$ values at the leaves. The likelihood is a function of the scaled branch lengths, denoted here as $\Theta$, and $\pi$, the unknown DAF at the root. The joint distribution $f\left(X_{i 1}, X_{i 2}, \ldots, X_{i J} \mid \Theta, \pi\right)$ is, in turn, an integral over the allele frequencies in the ancestral populations, represented as internal nodes in the tree. We approximate the integrals with sums by discretizing the allele frequencies. The discretized joint distribution is then obtained using a peeling algorithm (Felsenstein 1981), where the transition probabilities on each branch are given by the DAF as a function of the branch length (see File S1 for details). Because the SNPs are assumed to be independent, the full likelihood is a product over the SNPs

$$
L(\mathcal{D} ; \Theta, \pi)=\prod_{i=1}^{I} L\left(\mathcal{D}_{i} ; \Theta, \pi\right)
$$

Because SNP data contain only polymorphic sites, we further condition the preceding likelihood on polymorphic data as follows:

$$
L\left(\mathcal{D}_{i} ; \Theta, \pi \mid \text { polymorphism }_{i}\right)=\frac{L\left(\mathcal{D}_{i} ; \Theta, \pi\right)}{\mathbb{P}\left(\text { polymorphism }_{i} \mid \Theta, \pi\right)}
$$

where

$$
\mathbb{P}\left(\text { polymorphism }_{i} \mid \Theta, \pi\right)=1-L\left(\mathcal{D}_{i}^{0} ; \Theta, \pi\right)-L\left(\mathcal{D}_{i}^{1} ; \Theta, \pi\right)
$$

Here $\mathcal{D}_{i}^{0}$ and $\mathcal{D}_{i}^{1}$ are data corresponding to site $i$ where the allele was lost or fixed, respectively, in the samples from all populations

$$
\mathcal{D}_{i}^{0}=\left\{\left(0, n_{i j}\right) \mid 1 \leq j \leq J\right\}, \mathcal{D}_{i}^{1}=\left\{\left(n_{i j}, n_{i j}\right) \mid 1 \leq j \leq J\right\}
$$

We treat $\pi$, the root DAF, as a nuisance distribution, which we assume to be a beta distribution. For a given topology (i.e., tree without branch lengths), the most likely branch lengths and shape parameters of $\pi$ are recovered by numerically maximizing the likelihood conditional on polymorphism.

\section{Simulated data}

Using the topology depicted in Figure 2, we simulated multiple data sets containing independent SNPs under a WrightFisher model, given an ancenstral frequency $X_{i 5}$ sampled from $\pi$, the root DAF, which we set to be a beta distribution. We used two different scenarios, labeled I and II, summarized in Table 1. Scenario I has a uniform $\pi$ and large sample sizes, while scenario II is built to produce data that resemble the chimpanzee exome data analyzed later. For this, we used the chimpanzee sample sizes and scaled branch lengths and root DAF as inferred by the beta with spikes on the chimpanzee data (Table 2).

For each simulated data set, we estimated the branch lengths using both the beta and beta with spikes, as described previously. We additionally ran Kim Tree (Gautier and Vitalis 2013) using the default settings. Kim Tree is a method designed for inference of divergence times between populations evolving under pure genetic drift. It uses Kimura's solution to the diffusion limit for the DAF (Kimura 1955) and relies on a Bayesian Markov chain Monte Carlo (MCMC) approach. Here we use the posterior means of the branch lengths reported by this method as point estimates.

All methods estimated the branches leading to populations 1 and 2 well (Figure 3). Beta with spikes estimates the branch lengths more accurately and with lower variance than the beta approximation (Figure S1). Despite the fact that the spikes' probabilities do not perfectly match the true loss and fixation probabilities (Figure 1, E and F), this seems to have little effect on the accuracy of branch-length estimation for beta with spikes. For both scenarios, the branch leading to 
Table 1 Simulation study scenarios

\begin{tabular}{lcc}
\hline & Scenario I & Scenario II \\
\hline$(t / 2 N)_{4 \rightarrow 1}$ & $40 /(2 \cdot 200)=0.1$ & $132 /(2 \cdot 500)=0.132$ \\
$(t / 2 N)_{4 \rightarrow 2}$ & $40 /(2 \cdot 150)=0.133$ & $44 /(2 \cdot 500)=0.044$ \\
$(t / 2 N)_{5 \rightarrow 4}$ & $40 /(2 \cdot 150)=0.133$ & $14 /(2 \cdot 250)=0.028$ \\
$(t / 2 N)_{5 \rightarrow 3}$ & $80 /(2 \cdot 200)=0.2$ & $300 /(2 \cdot 250)=0.6$ \\
Shape parameters of $\pi$ & 1,1 & $0.0188,0.0195$ \\
Number of SNPs & 5000 & 10,000 \\
Sample sizes $n_{i 1}, n_{i 2}, n_{i 3}$ & $100,100,100$ & $22,24,12$ \\
Replicates & 50 & 50
\end{tabular}

This table indicates the values used for branch lengths $t$, population sizes $N$, and scaled branch lengths $t / 2 N$, shape parameters of the beta distribution $\pi$, the root DAF, and number of SNPs and sample sizes used in the two simulation scenarios.

population 2 and the inner branch from the root to population 4 have similar lengths, but the beta approximation and Kim Tree provide a worse estimate for the inner branch. This could be due to the fact that there are no data available resulting directly from the evolution on that branch, making the estimation problem harder. A similar result was obtained by Gautier and Vitalis (2013), who used trees with the same topology. Interestingly, beta with spikes recovers the inner branch much more accurately than either beta or Kim Tree. When measuring the accuracy of the inferred lengths as an average over all four branches (Table S1), it is clear that beta with spikes outperforms Kim Tree for both scenarios.

\section{Chimpanzee data}

The chimpanzee data analyzed here consisted of allele counts of autosomal synonymous SNPs obtained from exome sequencing of the Eastern, Central, and Western chimpanzee subspecies (Bataillon et al. 2015) for 11, 12, and 6 individuals, respectively. From the original data set containing 59,905 synonymous SNPs, we filtered the SNPs in which there were missing data, obtaining a total of 42,063 SNPs. We inferred the scaled branch lengths (Figure 4 and Table 2) using beta, beta with spikes, and Kim Tree on the full data set and on 50 smaller data sets containing only 10,000 randomly sampled SNPs. Beta with spikes and Kim Tree infer comparable branch lengths, with the exception of the branch leading to the Western chimpanzee subspecies. We additionally report in Table 2 the likelihood of the full data calculated using beta with spikes for the branch lengths inferred using the three methods and the ones reported in the original study (Bataillon et al. 2015). Bataillon et al. (2015) used an approximate Bayesian computation (ABC) approach to fit a demographic model to the synonymous SNPs. Their results are consistent with the results obtained here for the branches leading to the Eastern and Central chimpanzees. However, we obtained very different estimates for the remaining two branches. The likelihoods in Table 2 show that the branch lengths obtained by beta with spikes have the highest likelihood. This does not indicate which of the estimates is most correct because the methods use different modeling approaches. However, it does show that the differences between beta with spikes and beta/Kim Tree/ABC are a direct result of the modeling approach and not merely an artifact of the likelihood
Table 2 Inferred scaled branch lengths for the chimpanzee exome data

\begin{tabular}{lccccc}
\hline Method & $(t / 2 N)_{4 \rightarrow E}$ & $(t / 2 N)_{4 \rightarrow C}$ & $(t / 2 N)_{5 \rightarrow 4}$ & $(t / 2 N)_{5 \rightarrow W}$ & $\log L$ \\
\hline Beta & 0.273 & 0.086 & 0.002 & 0.955 & $-209,646$ \\
Beta with & 0.132 & 0.044 & 0.028 & 0.595 & $-204,045$ \\
$\quad$ spikes & & & & & \\
Kim Tree & 0.160 & 0.019 & 0.018 & 0.729 & $-205,838$ \\
ABC & 0.183 & 0.027 & 0.333 & 1.914 & $-233,802$ \\
\hline
\end{tabular}

${ }^{1}$ Bataillon et al. 2015

The notation follows that in Figure 2, and the populations correspond to those in Figure 4, with the leave's population: Eastern (E), Central (C), and Western (W). The last column shows the corresponding log likelihood calculated using beta with spikes.

numerical optimization being trapped in a local optimum. The discrepancy between the results of beta with spikes and those of $A B C$ is, perhaps, not surprising because the difference in inferred branch lengths seems to correlate with the goodness of fit of the $\mathrm{ABC}$ demographic model to the observed data. Bataillon et al. (2015) reported that their inferred demographic model shows a very good fit for the Central chimpanzees (absolute and relative difference in inferred branch length 0.017 and 0.386 , respectively), a slightly less good fit for the Eastern chimpanzees (absolute and relative difference in inferred branch length 0.051 and 0.386 , respectively), and a poorer fit for the Western chimpanzees (absolute and relative difference in inferred branch length 1.319 and 2.217, respectively).

\section{Data availability}

The beta, beta with spikes approximations, inference of divergence times, and simulation under a Wright-Fisher model were implemented in Python 2.7. The code is freely available at https://github.com/paula-tataru/SpikeyTree.

\section{Discussion}

We have developed a new approximation to the DAF as a function of time, conditional on an initial frequency, under a Wright-Fisher model with linear evolutionary pressures. Our work provides an accurate extension of the beta approximation (Balding and Nichols 1995, 1997; Sirén et al. 2011; Sirén 2012). As noted by Gautier and Vitalis (2013), the beta distribution ignores the possibility of loss or fixation of alleles. We addressed this issue by explicitly modeling the loss and fixation probabilities as two spikes at the boundaries. We showed that the addition of the spikes improves the quality of the approximation and results in more accurate inference of divergence times between populations that have been evolving under pure genetic drift. The DAF obtained as a solution of the diffusion equation is exactly the DAF expected under the Wright-Fisher model when the population size is large and the evolutionary pressures are not too strong, while the beta distribution is motivated only by the stationary DAF under linear evolutionary pressures. We therefore expected the beta with spikes to provide a less accurate approximation to the true DAF than the diffusion limit. Nevertheless, we 


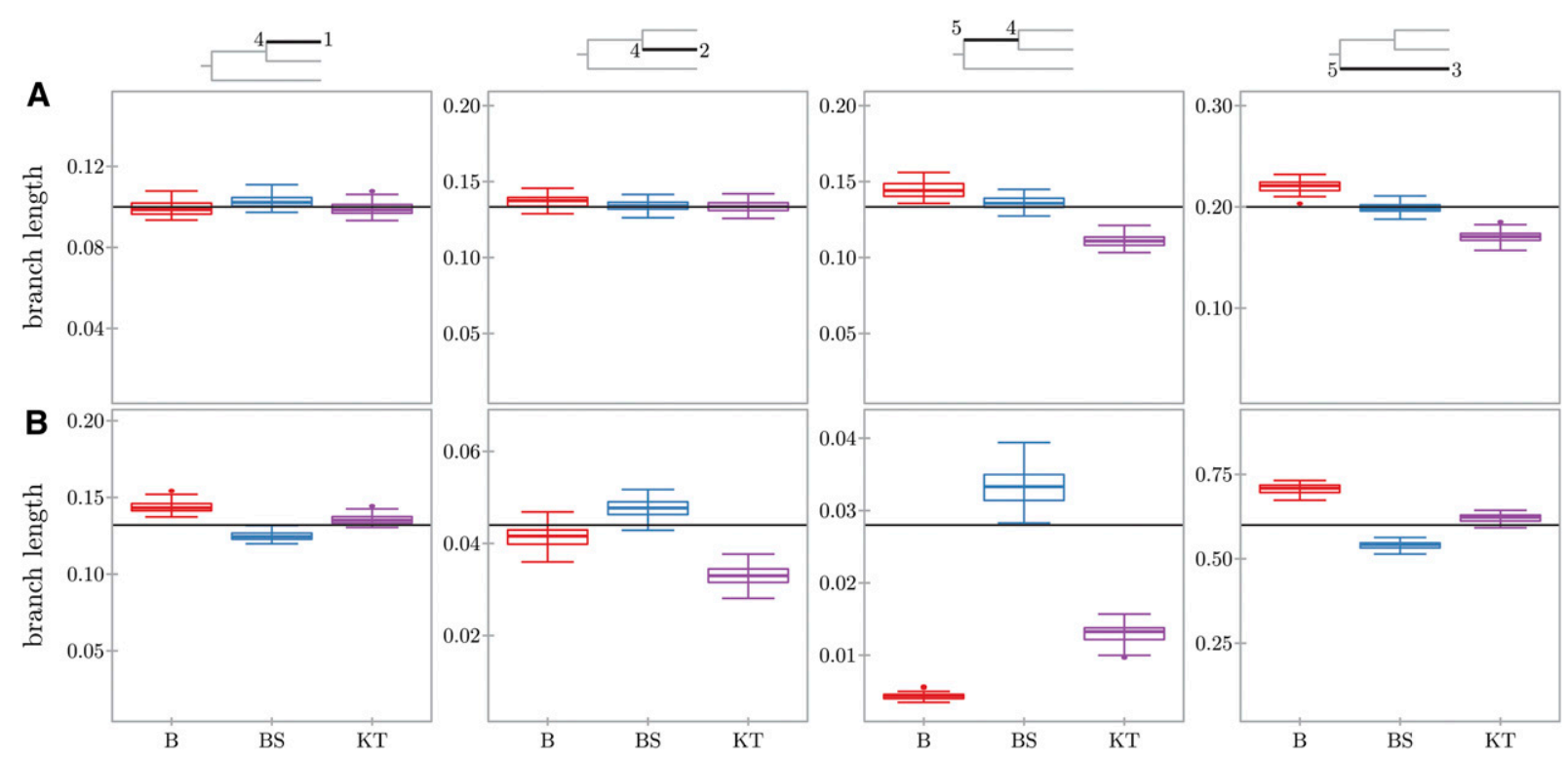

Figure 3 Inference of divergence times for simulation scenarios I (A) and II (B). The figure shows box plots summarizing the inferred lengths for the four branches of the tree, indicated at the top of each column in black. The inferred lengths are plotted for beta (B), beta with spikes (BS), and Kim Tree (KT) (Gautier and Vitalis 2013). The (true) simulated length of each branch is plotted as a horizontal line. Each plot is scaled relative to the corresponding simulated branch length $\tau$, with the limits of the $y$-axis set to $[\tau \cdot 0.1, \tau \cdot 1.5]$.

showed that our method can infer divergence times more accurately than Kim Tree (Gautier and Vitalis 2013), a software built for inference of divergence times using Kimura's solution to the diffusion limit (Kimura 1955). We would like to note here that the use of likelihood that is explicitly conditioning on polymorphic data [equation (13)] could potentially be the cause of beta with spikes outperforming Kim Tree.

\section{Computational complexity}

The advantage of beta with spikes becomes clearer when one considers its computational complexity. Diffusion methods rely on heavy computations, such as calculations of Gegenbauer polynomials (Gautier and Vitalis 2013), spectral decomposition of large matrices (Steinrücken et al. 2013, 2014), or matrix inverse (Zhao et al. 2013). In contrast, beta with spikes requires operations that are performed in constant time per iteration. Perhaps the most expensive evaluation is the beta function used in the loss and fixation probabilities, but very efficient approximations exist for this (Abramowitz and Stegun 1964). The difference in computational complexity is noticeable when comparing the running times of beta with spikes, implemented in Python 2.7, and

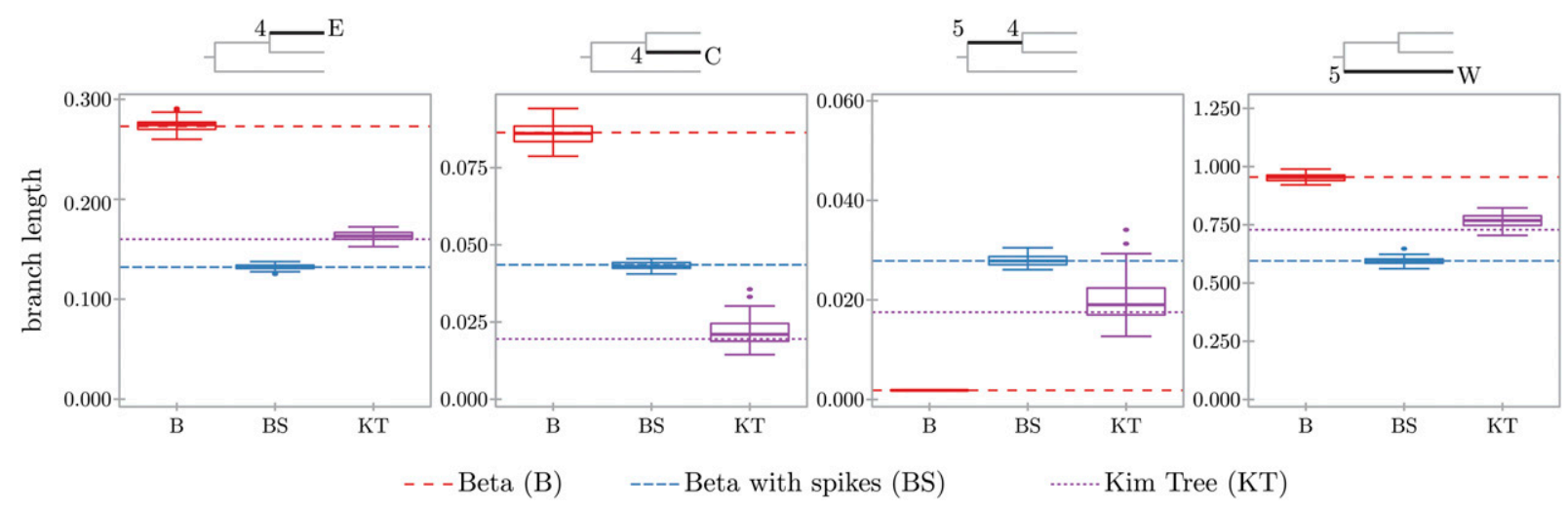

Figure 4 Inference of divergence times for the chimpanzee exome data. The figure shows box plots summarizing the inferred lengths using 50 data sets with 10,000 SNPs that were randomly sampled from the full data set. The corresponding tree branches are indicated at the top of each plot in black. The inferred lengths are plotted for beta (B), beta with spikes (BS), and Kim Tree (KT) (Gautier and Vitalis 2013). The nonsolid lines indicate the inferred lengths when running the methods on the full data set of 42,064 SNPs. The populations at the leaves are Eastern (E), Central (C), and Western (W). Each plot is scaled relative to the corresponding branch length $\tau$ inferred by beta with spikes on the full data set. The limits of the $y$-axis are set to $[\tau \cdot 0.05, \tau \cdot 1.5]$. 
Kim Tree, implemented in Fortran. For the chimpanzee data set of 42,063 SNPs, beta with spikes ran in just under $5 \mathrm{~min}$, while Kim Tree took almost an hour, even though Python 2.7 is a programming language that is less efficient than Fortran. We also note that the two inference methods are inherently different because here we used a numerical optimization procedure, while Kim Tree uses a Bayesian MCMC approach. Additionally, unlike Kim Tree, beta with spikes is a recursive method, and its accuracy and speed depend on the number of iterations performed (see File S1 for accuracy results for different numbers of iterations).

\section{Extensions}

We end this section by discussing possible extensions of the beta with spikes approximation and how these can be used in inference problems. Throughout this paper, we assumed that the population size is constant. Given its recursive formulation, beta with spikes lends itself naturally to incorporating variable population size without any increase in computational complexity. This can then be used for inference of population size backward in time, similar to methods relying on the coalescent with recombination (Li and Durbin 2011; Sheehan et al. 2013; Schiffels and Durbin 2014). A recently published method (Liu and Fu 2015) illustrates that allele frequency data, summarized as site frequency spectra, can be used efficiently for inference of variable population size backward in time. Even though Liu and Fu (2015) assumed that sites are independent and did not use linkage information, their method can handle larger data sets than the method of Li and Durbin (2011), which leads to more accurate inference of population sizes for the recent past. The results obtained by Liu and $\mathrm{Fu}$ (2015) indicate that beta with spikes could be used successfully for such demographic inference.

Another extension of the presented approximation would be to incorporate selection, which is a nonlinear evolutionary pressure. In the recent years, there has been a great focus on inference of selection coefficients from time-series data under a Wright-Fisher model (Malaspinas et al. 2012; Bank et al. 2014; Steinrücken et al. 2014; Foll et al. 2015; Terhorst et al. 2015). A newly developed statistical method aims at modeling the evolution of multilocus alleles under a Wright-Fisher model with selection (Terhorst et al. 2015) by fitting a multivariate normal distribution from the first moments of the DAF. Using the approach of Terhorst et al. (2015) for moment calculation, beta with spikes can be extended to nonlinear evolutionary pressures. Terhorst et al. (2015) did not treat the loss and fixation probabilities. However, because selection is expected to drive allele frequencies toward the boundaries faster than pure genetic drift, including the explicit spikes becomes crucial.

\section{Acknowledgments}

It is a pleasure to thank Thomas Mailund for helpful discussions. We also thank the two anonymous reviewers and the associate editor for their constructive suggestions and comments that helped to improve the manuscript. This work was supported, in part, by the European Research Council under the European Union's Seventh Framework Program (FP7/20072013, ERC grant number 311341) and the Danish Research Council (grant number DFF-400200382).

\section{Literature Cited}

Abramowitz, M., and I. A. Stegun, 1964 Handbook of Mathematical Functions: With Formulas, Graphs, and Mathematical Tables. Dover Publications, Mineola, NY.

Balding, D. J., and R. A. Nichols, 1995 A method for quantifying differentiation between populations at multiallelic loci and its implications for investigating identity and paternity. Genetica 96: 3-12.

Balding, D. J., and R. A. Nichols, 1997 Significant genetic correlations among Caucasians at forensic DNA loci. Heredity 78: 583-589.

Bank, C., G. B. Ewing, A. Ferrer-Admettla, M. Foll, and J. D. Jensen, 2014 Thinking too positive? Revisiting current methods of population genetic selection inference. Trends Genet. 30: 540546.

Bataillon, T., J. Duan, C. Hvilsom, X. Jin, Y. Li et al., 2015 Inference of purifying and positive selection in three subspecies of chimpanzees (Pan troglodytes) from exome sequencing. Genome Biol. Evol. 7: 1122-1132.

Coop, G., D. Witonsky, A. Di Rienzo, and J. K. Pritchard, 2010 Using environmental correlations to identify loci underlying local adaptation. Genetics 185: 1411-1423.

Crow, J., and M. Kimura, 1956 Some genetic problems in natural populations, pp. 1-22 in Proceedings of the Third Berkeley Symposium on Mathematical Statistics and Probability, Vol. 4. University of California Press, Oakland, CA.

Crow, J. F., and M. Kimura, 1970 An Introduction to Population Genetics Theory. Harper \& Row, New York.

Ewens, W. J., 2004 Mathematical Population Genetics 1: Theoretical Introduction, Vol. 27. Springer, New York.

Felsenstein, J., 1981 Evolutionary trees from DNA sequences: a maximum likelihood approach. J. Mol. Evol. 17: 368-376.

Foll, M., H. Shim, and J. D. Jensen, 2015 WFABC: a Wright-Fisher ABC-based approach for inferring effective population sizes and selection coefficients from time-sampled data. Mol. Ecol. Resour. 15: 87-98.

Gautier, M., T. D. Hocking, and J.-L. Foulley, 2010 A Bayesian outlier criterion to detect SNPs under selection in large data sets. PLoS One 5: e11913.

Gautier, M., and R. Vitalis, 2013 Inferring population histories using genome-wide allele frequency data. Mol. Biol. Evol. 30: 654-668.

Gudbjartsson, D. F., H. Helgason, S. A. Gudjonsson, F. Zink, A. Oddson et al., 2015 Large-scale whole-genome sequencing of the Icelandic population. Nat. Genet. 47: 435-444.

Hoban, S., G. Bertorelle, and O. E. Gaggiotti, 2012 Computer simulations: tools for population and evolutionary genetics. Nat. Rev. Genet. 13: 110-122.

Kimura, M., 1955 Solution of a process of random genetic drift with a continuous model. Proc. Natl. Acad. Sci. USA 41: 144.

Kimura, M., 1964 Diffusion models in population genetics. J. Appl. Probab. 1: 177-232.

Li, H., and R. Durbin, 2011 Inference of human population history from individual whole-genome sequences. Nature 475: 493496. 
Liu, X., and Y.-X. Fu, 2015 Exploring population size changes using SNP frequency spectra. Nat. Genet. 47: 555-559.

Malaspinas, A.-S., O. Malaspinas, S. N. Evans, and M. Slatkin, 2012 Estimating allele age and selection coefficient from time-serial data. Genetics 192: 599-607.

McKane, A., and D. Waxman, 2007 Singular solutions of the diffusion equation of population genetics. J. Theor. Biol. 247: 849858.

Nicholson, G., A. V. Smith, F. Jónsson, Ó. Gústafsson, K. Stefánsson et al., 2002 Assessing population differentiation and isolation from single-nucleotide polymorphism data. J. R. Stat. Soc. Series B Stat. Methodol. 64: 695-715.

Pickrell, J. K., and J. K. Pritchard, 2012 Inference of population splits and mixtures from genome-wide allele frequency data. PLoS Genet. 8: e1002967.

Romiguier, J., P. Gayral, M. Ballenghien, A. Bernard, V. Cahais et al., 2014 Comparative population genomics in animals uncovers the determinants of genetic diversity. Nature 515: 261263.

Rosenberg, N. A., and M. Nordborg, 2002 Genealogical trees, coalescent theory and the analysis of genetic polymorphisms. Nat. Rev. Genet. 3: 380-390.

Schiffels, S., and R. Durbin, 2014 Inferring human population size and separation history from multiple genome sequences. Nat. Genet. 46: 919-925.

Sheehan, S., K. Harris, and Y. S. Song, 2013 Estimating variable effective population sizes from multiple genomes: a sequentially Markov conditional sampling distribution approach. Genetics 194: 647-662.
Sirén, J., 2012 Statistical models for inferring the structure and history of populations from genetic data. Ph.D. thesis, University of Helsinki.

Sirén, J., P. Marttinen, and J. Corander, 2011 Reconstructing population histories from single nucleotide polymorphism data. Mol. Biol. Evol. 28: 673-683.

Song, Y. S., and M. Steinrücken, 2012 A simple method for finding explicit analytic transition densities of diffusion processes with general diploid selection. Genetics 190: 1117-1129.

Steinrücken, M., A. Bhaskar, and Y. S. Song, 2014 A novel spectral method for inferring general diploid selection from time series genetic data. Ann. Appl. Stat. 8: 2203.

Steinrücken, M., Y. R. Wang, and Y. S. Song, 2013 An explicit transition density expansion for a multiallelic Wright-Fisher diffusion with general diploid selection. Theor. Popul. Biol. 83: $1-14$.

Terhorst, J., C. Schlötterer, and Y. S. Song, 2015 Multilocus analysis of genomic time series data from experimental evolution. PLoS Genet. 11: e1005069.

Waxman, D., 2011 A compact result for the time-dependent probability of fixation at a neutral locus. J. Theor. Biol. 274: 131135.

Wright, S., 1945 The differential equation of the distribution of gene frequencies. Proc. Natl. Acad. Sci. USA 31: 382.

Zhao, L., X. Yue, and D. Waxman, 2013 Complete numerical solution of the diffusion equation of random genetic drift. Genetics 194: 973-985. 


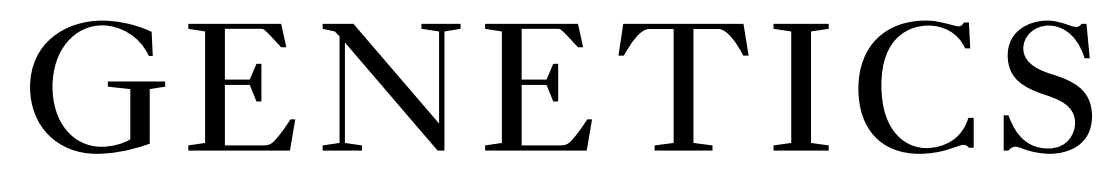

Supporting Information www.genetics.org/lookup/suppl/doi:10.1534/genetics.115.179606/-/DC1

\section{Inference Under a Wright-Fisher Model Using an Accurate Beta Approximation}

Paula Tataru, Thomas Bataillon, and Asger Hobolth 
Inference under a Wright-Fisher model

using an accurate beta approximation

\section{Supporting Material}

Paula Tataru*, Thomas Bataillon*, and Asger Hobolth*

\section{Contents}

Conditional mean and variance

Derivation of mean and variance of $X_{t}$

Derivation of loss and fixation probabilities of $X_{t}$

Approximation for small $a$ and $b \quad 7$

$\begin{array}{ll}\text { Approximation for large } N & 7\end{array}$

$\begin{array}{lr}\text { Parameter scaling } & 9\end{array}$

$\begin{array}{lr}\text { Discretization of beta and beta with spikes } & 9\end{array}$

$\begin{array}{ll}\text { Numerical accuracy of the beta and beta with spikes models } & 10\end{array}$

$\begin{array}{ll}\text { Likelihood calculation on a tree } & 10\end{array}$

$\begin{array}{ll}\text { Full data } & 11\end{array}$

$\begin{array}{ll}\text { Polymorphic data } & 12\end{array}$

$\begin{array}{ll}\text { The DAF at the root } & 13\end{array}$

$\begin{array}{ll}\text { Inference of divergence times: a simulation study } & 14\end{array}$

*Bioinformatics Research Centre, Aarhus University, Aarhus, 8000, Denmark 


\section{File S1}

\section{Conditional mean and variance}

If $X$ is a discrete random variable with values between 0 and 1 , its mean conditional on $X \notin\{0,1\}$ can be calculated as follows

$$
\begin{aligned}
\mathbb{E}[X \mid X \notin\{0,1\}] & =\sum_{x: x \notin\{0,1\}} x \cdot \mathbb{P}(X=x \mid X \notin\{0,1\}) \\
& =\sum_{x: x \notin\{0,1\}} x \cdot \frac{\mathbb{P}(X=x)}{\mathbb{P}(X \notin\{0,1\})} \\
& =\frac{1}{\mathbb{P}(X \notin\{0,1\})} \sum_{x: x \notin\{0,1\}} x \cdot \mathbb{P}(X=x) \\
& =\frac{1}{\mathbb{P}(X \notin\{0,1\})}(\mathbb{E}[X]-0 \cdot \mathbb{P}(X=0)-1 \cdot \mathbb{P}(X=1)) \\
& =\frac{\mathbb{E}[X]-\mathbb{P}(X=1)}{\mathbb{P}(X \notin\{0,1\})} .
\end{aligned}
$$

Similarly, we obtain

$$
\begin{aligned}
\mathbb{E}\left[X^{2} \mid X \notin\{0,1\}\right] & =\frac{\mathbb{E}\left[X^{2}\right]-\mathbb{P}(X=1)}{\mathbb{P}(X \notin\{0,1\})} \\
& =\frac{\operatorname{Var}(X)+\mathbb{E}[X]^{2}-\mathbb{P}(X=1)}{\mathbb{P}(X \notin\{0,1\})},
\end{aligned}
$$

from which

$$
\begin{aligned}
\operatorname{Var}(X \mid X \notin\{0,1\}) & =\mathbb{E}\left[X^{2} \mid X \notin\{0,1\}\right]-\mathbb{E}[X \mid X \notin\{0,1\}]^{2} \\
& =\frac{\operatorname{Var}(X)+\mathbb{E}[X]^{2}-\mathbb{P}(X=1)}{\mathbb{P}(X \notin\{0,1\})}-\mathbb{E}[X \mid X \notin\{0,1\}]^{2} .
\end{aligned}
$$

\section{Derivation of mean and variance of $X_{t}$}

To calculate the mean and variance of $X_{t}$ under the Wright-Fisher model, we rely on the laws of total mean and variance, respectively. Recall that $X_{t}=Z_{t} / 2 N$ and

$$
Z_{t+1} \mid Z_{t}=z_{t} \sim \operatorname{Bin}\left(2 N, g\left(x_{t}\right)\right)
$$

where $x_{t}=z_{t} / 2 N$. The evolutionary pressures $g(x)$ satisfy that $0 \leq g(x) \leq 1$ for all $0 \leq x \leq 1$. In the following, $g$ is a linear function in the allele frequency, $g(x)=(1-a) x+b$. 
The parameters $a$ and $b$ satisfy that $0 \leq b \leq a<1$ and typically, $a<<1$. We note that if $a=0$, then $b=0$. In the derivations below, we condition implicitly on $X_{0}=x_{0}$, population size $2 N$ and evolutionary pressures.

Let us start with the mean and variance of $X_{t+1}$ conditional on $X_{t}=x_{t}$, given by

$$
\begin{aligned}
\mathbb{E}\left[X_{t+1} \mid X_{t}=x_{t}\right] & =\frac{1}{2 N} \mathbb{E}\left[Z_{t+1} \mid Z_{t}=z_{t}\right] \\
& =\frac{1}{2 N} 2 N g\left(x_{t}\right) \\
& =g\left(x_{t}\right), \\
\operatorname{Var}\left(X_{t+1} \mid X_{t}=x_{t}\right) & =\frac{1}{4 N^{2}} \operatorname{Var}\left(Z_{t+1} \mid Z_{t}=z_{t}\right) \\
& =\frac{1}{4 N^{2}} 2 N g\left(x_{t}\right)\left(1-g\left(x_{t}\right)\right) \\
& =\frac{1}{2 N} g\left(x_{t}\right)\left(1-g\left(x_{t}\right)\right) .
\end{aligned}
$$

First, using the law of total expectation, we have that

$$
\begin{aligned}
\mathbb{E}\left[X_{t}\right] & =\mathbb{E}\left[\mathbb{E}\left[X_{t} \mid X_{t-1}\right]\right] \\
& =\mathbb{E}\left[g\left(X_{t-1}\right)\right] \\
& =\mathbb{E}\left[(1-a) X_{t-1}+b\right] \\
& =(1-a) \mathbb{E}\left[X_{t-1}\right]+b \\
& =(1-a) \mathbb{E}\left[\mathbb{E}\left[X_{t-1} \mid X_{t-2}\right]\right]+b \\
& =\ldots \\
& =(1-a)^{t} x_{0}+b \sum_{i=0}^{t-1}(1-a)^{i} .
\end{aligned}
$$

When $a=b=0$, the mean becomes

$$
\mathbb{E}\left[X_{t}\right]=x_{0}
$$

If $a \neq 0$,

$$
\sum_{i=0}^{t-1}(1-a)^{i}=\frac{1-(1-a)^{t}}{a},
$$


and this gives

$$
\mathbb{E}\left[X_{t}\right]=\frac{b}{a}+(1-a)^{t}\left(x_{0}-\frac{b}{a}\right)
$$

We use a similar approach to determine the variance of $X_{t}$, this time relying on the law of total variance

$$
\begin{aligned}
\operatorname{Var}\left(X_{t}\right) & =\mathbb{E}\left[\operatorname{Var}\left(X_{t} \mid X_{t-1}\right)\right]+\operatorname{Var}\left(\mathbb{E}\left[X_{t} \mid X_{t-1}\right]\right) \\
& =\mathbb{E}\left[\frac{1}{2 N} g\left(X_{t-1}\right)\left(1-g\left(X_{t-1}\right)\right)\right]+\operatorname{Var}\left(g\left(X_{t-1}\right)\right) \\
& =\frac{1}{2 N} \mathbb{E}\left[g\left(X_{t-1}\right)\right]-\frac{1}{2 N} \mathbb{E}\left[g\left(X_{t-1}\right)^{2}\right]+\operatorname{Var}\left(g\left(X_{t-1}\right)\right) \\
& =\frac{1}{2 N} \mathbb{E}\left[g\left(X_{t-1}\right)\right]-\frac{1}{2 N} \operatorname{Var}\left(g\left(X_{t-1}\right)\right)-\frac{1}{2 N} \mathbb{E}\left[g\left(X_{t-1}\right)\right]^{2}+\operatorname{Var}\left(g\left(X_{t-1}\right)\right) \\
& =\frac{1}{2 N} \mathbb{E}\left[g\left(X_{t-1}\right)\right]\left(1-\mathbb{E}\left[g\left(X_{t-1}\right)\right]\right)+\left(1-\frac{1}{2 N}\right) \operatorname{Var}\left(g\left(X_{t-1}\right)\right) \\
& =\frac{1}{2 N} \mathbb{E}\left[X_{t}\right]\left(1-\mathbb{E}\left[X_{t}\right]\right)+\left(1-\frac{1}{2 N}\right)(1-a)^{2} \operatorname{Var}\left(X_{t-1}\right) .
\end{aligned}
$$

Iterating the above,

$$
\operatorname{Var}\left(X_{t}\right)=\frac{1}{2 N} \sum_{i=1}^{t}(1-a)^{2(t-i)}\left(1-\frac{1}{2 N}\right)^{t-i} \mathbb{E}\left[X_{i}\right]\left(1-\mathbb{E}\left[X_{i}\right]\right) .
$$

Let us observe that, for any $c$,

$$
\begin{aligned}
\frac{1}{2 N} \sum_{i=1}^{t}(1-a)^{c(t-i)}\left(1-\frac{1}{2 N}\right)^{t-i} & =\frac{1}{2 N} \cdot \frac{1-(1-a)^{c t}\left(1-\frac{1}{2 N}\right)^{t}}{1-(1-a)^{c}\left(1-\frac{1}{2 N}\right)} \\
& =\frac{1-(1-a)^{c t}\left(1-\frac{1}{2 N}\right)^{t}}{2 N-(1-a)^{c}(2 N-1)} .
\end{aligned}
$$

When $a=b=0$ and using $c=0$ in the above, the variance becomes

$$
\operatorname{Var}\left(X_{t}\right)=x_{0}\left(1-x_{0}\right)\left[1-\left(1-\frac{1}{2 N}\right)^{t}\right] \text {. }
$$

If $a \neq 0$,

$$
\begin{aligned}
\mathbb{E}\left[X_{i}\right]\left(1-\mathbb{E}\left[X_{i}\right]\right)=\frac{b}{a} & \left(1-\frac{b}{a}\right) \\
& +\left(1-\frac{2 b}{a}\right)(1-a)^{i}\left(x_{0}-\frac{b}{a}\right) \\
& -(1-a)^{2 i}\left(x_{0}-\frac{b}{a}\right)^{2}
\end{aligned}
$$


and using $c=1$ and $c=2$, respectively, we obtain the variance

$$
\begin{aligned}
\operatorname{Var}\left(X_{t}\right)=\frac{b}{a} & \left(1-\frac{b}{a}\right) \frac{1}{2 N} \sum_{i=1}^{t}(1-a)^{2(t-i)}\left(1-\frac{1}{2 N}\right)^{t-i} \\
& +\left(1-\frac{2 b}{a}\right)\left(x_{0}-\frac{b}{a}\right)(1-a)^{t} \frac{1}{2 N} \sum_{i=1}^{t}(1-a)^{t-i}\left(1-\frac{1}{2 N}\right)^{t-i} \\
& -\left(x_{0}-\frac{b}{a}\right)^{2}(1-a)^{2 t} \frac{1}{2 N} \sum_{i=1}^{t}\left(1-\frac{1}{2 N}\right)^{t-i} \\
=\frac{b}{a} & \left(1-\frac{b}{a}\right) \frac{1-(1-a)^{2 t}\left(1-\frac{1}{2 N}\right)^{t}}{2 N-(1-a)^{2}(2 N-1)} \\
& +\left(1-\frac{2 b}{a}\right)\left(x_{0}-\frac{b}{a}\right)(1-a)^{t} \frac{1-(1-a)^{t}\left(1-\frac{1}{2 N}\right)^{t}}{2 N-(1-a)(2 N-1)} \\
& -\left(x_{0}-\frac{b}{a}\right)^{2}(1-a)^{2 t}\left(1-\left(1-\frac{1}{2 N}\right)^{t}\right) .
\end{aligned}
$$

See the parameter scaling section for a comparison with the derivations obtained by Sirén (2012). We note that Sirén (2012) relies on approximations resulting from the infinite population limit, while the above equations hold for any population size.

The derivations for the mean and variance use the linearity of the evolutionary pressures through the simplification that

$$
\begin{aligned}
\mathbb{E}\left[(1-a) X_{t}+b\right] & =(1-a) \mathbb{E}\left[X_{t}\right]+b, \\
\operatorname{Var}\left((1-a) X_{t}+b\right) & =(1-a)^{2} \operatorname{Var}\left(X_{t}\right) .
\end{aligned}
$$

When $g(x)$ is a polynomial of higher order, such as in the case of selection, the derivation requires higher moments of $X_{t}$, leading to an explosion in the moments needed and rendering the above approach untractable in such situations.

\section{Derivation of loss and fixation probabilities of $X_{t}$}

To determine $\mathbb{P}\left(X_{t+1}=0\right)$ and $\mathbb{P}\left(X_{t+1}=1\right)$, we use the law of total probability in an

approach similar to the above. Additionally, we rely on the approximation that $X_{t}$ follows 
a known $f_{B}^{\star}$ beta with spikes distribution to obtain

$$
\begin{aligned}
\mathbb{P}\left(X_{t+1}=0\right)= & \int_{0}^{1} \mathbb{P}\left(X_{t+1}=0 \mid X_{t}=x\right) \cdot f_{B}^{\star}(x ; t) \mathrm{d} x \\
= & \mathbb{P}\left(X_{t+1}=0 \mid X_{t}=0\right) \cdot \mathbb{P}\left(X_{t}=0\right)+\mathbb{P}\left(X_{t+1}=0 \mid X_{t}=1\right) \cdot \mathbb{P}\left(X_{t}=1\right) \\
& \quad+\mathbb{P}\left(X_{t} \notin\{0,1\}\right) \cdot \int_{0}^{1} \mathbb{P}\left(X_{t+1}=0 \mid X_{t}=x\right) \cdot \frac{x^{\alpha_{t}^{\star}-1}(1-x)^{\beta_{t}^{\star}-1}}{\mathrm{~B}\left(\alpha_{t}^{\star}, \beta_{t}^{\star}\right)} \mathrm{d} x \\
= & \mathbb{P}\left(X_{t}=0\right) \cdot(1-g(0))^{2 N}+\mathbb{P}\left(X_{t}=1\right) \cdot(1-g(1))^{2 N} \\
& \quad+\mathbb{P}\left(X_{t} \notin\{0,1\}\right) \cdot \int_{0}^{1}(1-g(x))^{2 N} \cdot \frac{x^{\alpha_{t}^{\star}-1}(1-x)^{\beta_{t}^{\star}-1}}{\mathrm{~B}\left(\alpha_{t}^{\star}, \beta_{t}^{\star}\right)} \mathrm{d} x,
\end{aligned}
$$

where $\mathrm{B}(\alpha, \beta)$ is the beta function.

To calculate the above integral for linear evolutionary pressures, we rely on the hypergeometric function. Let ${ }_{2} F_{1}(-m, b ; c ; z)$ ((Erdélyi et al. 1953), 2.1.3) be the hypergeometric function for $m \in \mathbb{N}, c, d \in \mathbb{R}_{+}$and $z \in \mathbb{R}$, given by

$$
{ }_{2} F_{1}(-m, c ; c+d ; z)=\frac{1}{\mathrm{~B}(c, d)} \int_{0}^{1} x^{c-1}(1-x)^{d-1}(1-z x)^{m} \mathrm{~d} x \text {. }
$$

We have that (recall that $0 \leq b<1$ )

$$
\begin{aligned}
\int_{0}^{1}( & 1-g(x))^{2 N} \cdot \frac{x^{\alpha_{t}^{\star}-1}(1-x)^{\beta_{t}^{\star}-1}}{\mathrm{~B}\left(\alpha_{t}^{\star}, \beta_{t}^{\star}\right)} \mathrm{d} x \\
= & \frac{1}{\mathrm{~B}\left(\alpha_{t}^{\star}, \beta_{t}^{\star}\right)} \int_{0}^{1}((1-b)-(1-a) x)^{2 N} x^{\alpha_{t}^{\star}-1}(1-x)^{\beta_{t}^{\star}-1} \mathrm{~d} x \\
= & \frac{(1-b)^{2 N}}{\mathrm{~B}\left(\alpha_{t}^{\star}, \beta_{t}^{\star}\right)} \int_{0}^{1}\left(1-\frac{1-a}{1-b} x\right)^{2 N} x^{\alpha_{t}^{\star}-1}(1-x)^{\beta_{t}^{\star}-1} \mathrm{~d} x \\
= & (1-b)^{2 N}{ }_{2} F_{1}\left(-2 N, \alpha_{t}^{\star} ; \alpha_{t}^{\star}+\beta_{t}^{\star} ; \frac{1-a}{1-b}\right),
\end{aligned}
$$

leading to the full expression for the loss probability

$$
\begin{aligned}
\mathbb{P}\left(X_{t+1}=0\right)=\mathbb{P}( & \left.X_{t}=0\right) \cdot(1-b)^{2 N}+\mathbb{P}\left(X_{t}=1\right) \cdot(a-b)^{2 N} \\
& +\mathbb{P}\left(X_{t} \notin\{0,1\}\right) \cdot(1-b)^{2 N} \cdot{ }_{2} F_{1}\left(-2 N, \alpha_{t}^{\star} ; \alpha_{t}^{\star}+\beta_{t}^{\star} ; \frac{1-a}{1-b}\right)
\end{aligned}
$$


Similarly, for $b \neq 0$ (if $b=0$, see below), we obtain the fixation probability

$$
\begin{aligned}
\mathbb{P}\left(X_{t+1}=1\right)=\mathbb{P} & \left(X_{t}=0\right) \cdot b^{2 N}+\mathbb{P}\left(X_{t}=1\right) \cdot(1-a+b)^{2 N} \\
& +\mathbb{P}\left(X_{t} \notin\{0,1\}\right) \cdot b^{2 N} \cdot{ }_{2} F_{1}\left(-2 N, \alpha_{t}^{\star} ; \alpha_{t}^{\star}+\beta_{t}^{\star} ;-\frac{1-a}{b}\right) .
\end{aligned}
$$

Approximation for small $a$ and $b$ The hypergeometric function can be cumbersome and slow to evaluate. Typically the parameters $a$ and $b$ are small and we can use that

$$
\begin{array}{rlrl}
1-g(x) & =(1-a)(1-x)+a-b & & \approx(1-a)(1-x), \\
g(x) & =(1-a) x+b & \approx(1-a) x
\end{array}
$$

to more easily reduce the above integrals to

$$
\begin{aligned}
\int_{0}^{1}(1-g(x))^{2 N} \cdot \frac{x^{\alpha_{t}^{\star}-1}(1-x)^{\beta_{t}^{\star}-1}}{\mathrm{~B}\left(\alpha_{t}^{\star}, \beta_{t}^{\star}\right)} \mathrm{d} x & \approx(1-a)^{2 N} \cdot \int_{0}^{1} \frac{x^{\alpha_{t}^{\star}-1}(1-x)^{\beta_{t}^{\star}+2 N-1}}{\mathrm{~B}\left(\alpha_{t}^{\star}, \beta_{t}^{\star}\right)} \mathrm{d} x \\
& =(1-a)^{2 N} \cdot \frac{\mathrm{B}\left(\alpha_{t}^{\star}, \beta_{t}^{\star}+2 N\right)}{\mathrm{B}\left(\alpha_{t}^{\star}, \beta_{t}^{\star}\right)}, \\
\int_{0}^{1} g(x)^{2 N} \cdot \frac{x^{\alpha_{t}^{\star}-1}(1-x)^{\beta_{t}^{\star}-1}}{\mathrm{~B}\left(\alpha_{t}^{\star}, \beta_{t}^{\star}\right)} \mathrm{d} x & \approx(1-a)^{2 N} \cdot \frac{\mathrm{B}\left(\alpha_{t}^{\star}+2 N, \beta_{t}^{\star}\right)}{\mathrm{B}\left(\alpha_{t}^{\star}, \beta_{t}^{\star}\right)},
\end{aligned}
$$

from which

$$
\begin{aligned}
\mathbb{P}\left(X_{t+1}=0\right) \approx & \mathbb{P}\left(X_{t}=0\right) \cdot(1-b)^{2 N}+\mathbb{P}\left(X_{t}=1\right) \cdot(a-b)^{2 N} \\
& +\mathbb{P}\left(X_{t} \notin\{0,1\}\right) \cdot(1-a)^{2 N} \cdot \frac{\mathrm{B}\left(\alpha_{t}^{\star}, \beta_{t}^{\star}+2 N\right)}{\mathrm{B}\left(\alpha_{t}^{\star}, \beta_{t}^{\star}\right)}, \\
\mathbb{P}\left(X_{t+1}=1\right) \approx & \mathbb{P}\left(X_{t}=0\right) \cdot b^{2 N}+\mathbb{P}\left(X_{t}=1\right) \cdot(1-a+b)^{2 N} \\
& +\mathbb{P}\left(X_{t} \notin\{0,1\}\right) \cdot(1-a)^{2 N} \cdot \frac{\mathrm{B}\left(\alpha_{t}^{\star}+2 N, \beta_{t}^{\star}\right)}{\mathrm{B}\left(\alpha_{t}^{\star}, \beta_{t}^{\star}\right)} .
\end{aligned}
$$

For the results reported in the main text and below (in numerical accuracy and inference of divergence times sections), we used the above approximation for small $a$ and $b$.

Approximation for large $N$ A widely used assumption in the derivations based on the Wright-Fisher model, such as the diffusion limit, is that the population size $N$ is large, and $a$ and $b$ are small such that

$$
\lim _{N \rightarrow \infty} 2 N a=A, \quad \quad \lim _{N \rightarrow \infty} 2 N b=B .
$$


Additionally, the time is scaled by the population size, $\tau=t / 2 N$. We set $\Delta=1 / 2 N$. Because $a$ and $b$ are small, we build on the previous approximation.

Let $\Gamma(c)$ be the Gamma function and note that, for large $N$ ((Erdélyi et al. 1953), 1.18),

$$
\frac{\Gamma(N+c)}{\Gamma(N+c+d)} \approx\left(\frac{1}{N}\right)^{d}\left(1-\frac{d(c+2 d-1)}{2 N}\right) .
$$

We then have

$$
\begin{aligned}
\frac{\mathrm{B}\left(\alpha_{t}^{\star}, \beta_{t}^{\star}+2 N\right)}{\mathrm{B}\left(\alpha_{t}^{\star}, \beta_{t}^{\star}\right)} & =\frac{\Gamma\left(\alpha_{t}^{\star}\right) \Gamma\left(\beta_{t}^{\star}+2 N\right)}{\Gamma\left(\alpha_{t}^{\star}+\beta_{t}^{\star}+2 N\right)} \cdot \frac{\Gamma\left(\alpha_{t}^{\star}+\beta_{t}^{\star}\right)}{\Gamma\left(\alpha_{t}^{\star}\right) \Gamma\left(\beta_{t}^{\star}\right)} \\
& =\frac{\Gamma\left(\alpha_{t}^{\star}+\beta_{t}^{\star}\right)}{\Gamma\left(\beta_{t}^{\star}\right)} \cdot \frac{\Gamma\left(2 N+\beta_{t}^{\star}\right)}{\Gamma\left(2 N+\alpha_{t}^{\star}+\beta_{t}^{\star}\right)} \\
& \approx \frac{\Gamma\left(\alpha_{t}^{\star}+\beta_{t}^{\star}\right)}{\Gamma\left(\beta_{t}^{\star}\right)} \cdot\left(\frac{1}{2 N}\right)^{\alpha_{t}^{\star}} \cdot\left(1-\frac{\alpha_{t}^{\star}\left(2 \alpha_{t}^{\star}+\beta_{t}^{\star}-1\right)}{4 N}\right) \\
& =\frac{\Gamma\left(\alpha_{t}^{\star}+\beta_{t}^{\star}\right)}{\Gamma\left(\beta_{t}^{\star}\right)} \cdot \Delta^{\alpha_{t}^{\star}} \cdot\left(1-\frac{1}{2} \Delta \alpha_{t}^{\star}\left(2 \alpha_{t}^{\star}+\beta_{t}^{\star}-1\right)\right),
\end{aligned}
$$

and, similarly,

$$
\frac{\mathrm{B}\left(\alpha_{t}^{\star}+2 N, \beta_{t}^{\star}\right)}{\mathrm{B}\left(\alpha_{t}^{\star}, \beta_{t}^{\star}\right)} \approx \frac{\Gamma\left(\alpha_{t}^{\star}+\beta_{t}^{\star}\right)}{\Gamma\left(\alpha_{t}^{\star}\right)} \cdot \Delta^{\beta_{t}^{\star}} \cdot\left(1-\frac{1}{2} \Delta \beta_{t}^{\star}\left(\alpha_{t}^{\star}+2 \beta_{t}^{\star}-1\right)\right) .
$$

Using that

$$
\begin{aligned}
\lim _{N \rightarrow \infty}(1-a)^{2 N} & =e^{-A}, & \lim _{N \rightarrow \infty}(1-b)^{2 N} & =e^{-B}, \\
\lim _{N \rightarrow \infty}(1-a+b)^{2 N} & =e^{-(A-B)}, & \lim _{N \rightarrow \infty}(a-b)^{2 N} & =0,
\end{aligned}
$$

we obtain the recursion in scaled time for loss and fixation probabilities to be

$$
\begin{aligned}
\mathbb{P}\left(X_{\tau+\Delta}=0\right) \approx & \mathbb{P}\left(X_{\tau}=0\right) \cdot e^{-B} \\
& +\mathbb{P}\left(X_{\tau} \notin\{0,1\}\right) \cdot e^{-A} \cdot \frac{\Gamma\left(\alpha_{\tau}^{\star}+\beta_{\tau}^{\star}\right)}{\Gamma\left(\beta_{\tau}^{\star}\right)} \cdot \Delta^{\alpha_{\tau}^{\star}} \cdot\left(1-\frac{1}{2} \Delta \alpha_{\tau}^{\star}\left(2 \alpha_{\tau}^{\star}+\beta_{\tau}^{\star}-1\right)\right), \\
\mathbb{P}\left(X_{\tau+\Delta}=1\right) \approx & \mathbb{P}\left(X_{\tau}=1\right) \cdot e^{-(A-B)} \\
& +\mathbb{P}\left(X_{\tau} \notin\{0,1\}\right) \cdot e^{-A} \cdot \frac{\Gamma\left(\alpha_{\tau}^{\star}+\beta_{\tau}^{\star}\right)}{\Gamma\left(\alpha_{\tau}^{\star}\right)} \cdot \Delta^{\beta_{\tau}^{\star}} \cdot\left(1-\frac{1}{2} \Delta \beta_{\tau}^{\star}\left(\alpha_{\tau}^{\star}+2 \beta_{\tau}^{\star}-1\right)\right) .
\end{aligned}
$$




\section{Parameter scaling}

As noted above, one common assumption is that the population size $N$ is large and $a$ and $b$ are small. One central result of the diffusion limit is that the allele frequency distribution is entirely determined by the scaled time $\tau=t / 2 \mathrm{~N}$ and parameters $A=2 \mathrm{Na}$ and $\mathrm{B}=2 \mathrm{Nb}$ (Ewens 2004). The same holds for the beta distribution. Using that

$$
\begin{array}{rlrl}
(1-a)^{t} & \approx e^{-A \tau}, & 2 N-(1-a)(2 N-1) & \approx 1+A, \\
\left(1-\frac{1}{2 N}\right)^{t} \approx e^{-\tau}, & 2 N-(1-a)^{2}(2 N-1) & \approx 1+2 A,
\end{array}
$$

we obtain the mean and variance as a function of the scaled parameters to be

$$
\begin{gathered}
\mathbb{E}\left[X_{\tau}\right]= \begin{cases}x_{0} & \text { if } a=b=0, \\
\frac{B}{A}+e^{-A \tau}\left(x_{0}-\frac{B}{A}\right) & \text { otherwise, }\end{cases} \\
\operatorname{Var}\left(X_{\tau}\right)=\left\{\begin{array}{cc}
x_{0}\left(1-x_{0}\right)\left(1-e^{-\tau}\right) & \text { if } a=b=0, \\
\frac{B}{A}\left(1-\frac{B}{A}\right) \frac{1-e^{-(2 A+1) \tau}}{1+2 A} & \text { otherwise. } \\
+\left(1-\frac{2 B}{A}\right)\left(x_{0}-\frac{B}{A}\right) e^{-A \tau} \frac{1-e^{-(A+1) \tau}}{1+A} & \\
-\left(x_{0}-\frac{B}{A}\right)^{2} e^{-2 A \tau}\left(1-e^{-\tau}\right) &
\end{array}\right.
\end{gathered}
$$

The above equations are equivalent to the ones by Sirén (2012) (up to some minor typographical errors, as confirmed by correspondence with the author).

The same property holds for the beta with spikes, as shown in the above derivation for large $N$, where the loss and fixation probability are written as functions of the scaled parameters.

\section{Discretization of beta and beta with spikes}

For the presented results, the beta and beta with spikes distributions need to be discretized in $K+1$ bins. We chose bins that, except for the first and last bin, are centered around $\frac{k}{K}$ for $1 \leq k \leq K-1$, given by

$$
\left[0, \frac{1}{2 K}\right],\left[\frac{1}{2 K}, \frac{3}{2 K}\right], \ldots,\left[\frac{2 k-1}{2 K}, \frac{2 k+1}{2 K}\right], \ldots,\left[\frac{2 K-3}{2 K}, \frac{2 K-1}{2 K}\right],\left[\frac{2 K-1}{2 K}, 1\right],
$$


Next to the $K+1$ probabilities corresponding to each bin, the beta with spikes distribution contains two extra spike probabilities for 0 and 1.

\section{Numerical accuracy of the beta and beta with spikes models}

To investigate how well the beta with spikes approximates the true distribution of allele frequency (DAF) and, in particular, if it provides a better approximation than the beta distribution, we compared the two with the DAF calculated directly from the Wright-Fisher. For this purpose, we discretized the approximated distributions using $K=2 N$. This leads to a unique mapping between the true discrete allele frequencies $k / 2 N, 0 \leq k \leq 2 N$ and the bins. As the first and last bins correspond to frequencies 0 and 1 , respectively, and the beta with spikes contains explicit probabilities for these two frequencies, we merged the first and last two bins to $\left[0, \frac{3}{4 N}\right]$ and $\left[\frac{4 N-3}{4 N}, 1\right]$ for calculating the discrete probability for frequencies $\frac{1}{2 N}$ and $\frac{2 N-1}{2 N}$, respectively.

We used a population size $2 N=200$ and for a range of initial frequencies $x_{0}$, times $t$ and parameters $a$ and $b$, we calculated the Hellinger distance between the true and approximated distributions. For two discrete distributions $P=\left(p_{1}, \ldots, p_{k}\right)$ and $Q=\left(q_{1}, \ldots, q_{k}\right)$, the Hellinger distance is given by

$$
h(P, Q)=\frac{1}{\sqrt{2}} \sqrt{\sum_{i=1}^{k}\left(\sqrt{p_{i}}-\sqrt{q_{i}}\right)^{2}} .
$$

The Hellinger distance lies between 0 and 1, with 0 indicating perfect match between the two distributions, while the value of 1 is achieved when $P$ assigns probability zero to every set where $Q$ assigns a positive probability, and vice versa. The Hellinger distance for the beta and beta with spikes is given in Figure 1 in the main text.

\section{Likelihood calculation on a tree}

The probability of observing the data for a given tree is a function of the scaled branch lengths, denoted here as $\Theta$, and $\pi$, the DAF at the root. We are interested in calculating 
the likelihood $L(\mathcal{D} ; \Theta, \pi)$ of the data $\mathcal{D}=\left\{\left(z_{i j}, n_{i j}\right) \mid 1 \leq i \leq I, 1 \leq j \leq J\right\}$, for $I$ SNPs in $J$ populations. The SNPs are assumed to be realizations of independent and identically distributed random variables (of dimension $J$ ). Then the full likelihood of the data can be written as a product over the independent sites

$$
L(\mathcal{D} ; \Theta, \pi)=\prod_{i=1}^{I} L\left(\mathcal{D}_{i} ; \Theta, \pi\right),
$$

where $\mathcal{D}_{i}=\left\{\left(z_{i j}, n_{i j}\right) \mid 1 \leq j \leq J\right\}$ is the observed data for SNP $i$. We therefore present below how to calculate the likelihood for one SNP and, for notation simplicity, drop the index $i$.

Full data Assuming Hardy-Weinberg equilibrium, the probability of observing $z$ alleles in a sample of size $n$, given the population allele frequency $x$, follows from the binomial distribution

$$
\mathbb{P}(z \mid n, x)=\left(\begin{array}{l}
n \\
z
\end{array}\right) x^{z}(1-x)^{n-z}
$$

However, the allele frequencies $x_{j}, 1 \leq j \leq J$, are unobserved and the likelihood of the data is obtained by integrating over the unknown allele frequencies

$$
\begin{array}{r}
L(\mathcal{D} ; \Theta, \pi)=\int_{0}^{1} \int_{0}^{1} \ldots \int_{0}^{1} f\left(X_{1}, X_{2}, \ldots, X_{J} \mid \Theta, \pi\right) \\
\cdot \prod_{j=1}^{J} \mathbb{P}\left(z_{j} \mid n_{j}, X_{j}\right) \mathrm{d} X_{1} \mathrm{~d} X_{2} \ldots \mathrm{d} X_{J},
\end{array}
$$

where $f\left(X_{1}, X_{2}, \ldots, X_{J} \mid \Theta, \pi\right)$ is the joint distribution of the $X_{j}$ 's at the leaves. The joint distribution is, in turn, an integral over the allele frequencies in the ancestral populations, represented as internal nodes in the tree. To calculate the likelihood and the joint distribution, we discretize the allele frequencies in $K+1$ bins as detailed above. Let bin number $0 \leq k \leq K$ from before be $\left[l_{k}, u_{k}\right]$ (i.e. $l_{k}=\max \{0,2 k-1\} / 2 K$ and $\left.u_{k}=\min \{2 k+1,1\} / 2 K\right)$. Then, for each branch length $t / 2 N$ we can calculate the discrete transition probabilities as

$$
\mathbb{P}\left(X_{j} \in\left[l_{k}, u_{k}\right] \mid X_{l}=k_{0} / K, t / 2 N\right)=\int_{l_{k}}^{u_{k}} f\left(x ; k_{0} / K, t, N\right) \mathrm{d} x
$$


where $f\left(x ; k_{0} / K, t, N\right)$ is the DAF over $t$ generations in a population of size $2 N$, conditional on a initial frequency $k_{0} / K, 0 \leq k_{0} \leq K$. The distribution $f$ is replaced by either $f_{B}$ for the beta, or $f_{B}^{\star}$ for the beta with spikes. When using the beta with spikes, we use two additional probabilities for $X_{j}=0$ and $X_{j}=1$. With these transition probabilities at hand, we can efficiently calculate the joint distribution using a peeling algorithm (Felsenstein 1981).

For the tree depicted in Figure $2, \Theta=\left((t / 2 N)_{5 \rightarrow 3},(t / 2 N)_{5 \rightarrow 4},(t / 2 N)_{4 \rightarrow 1},(t / 2 N)_{4 \rightarrow 2}\right)$ and conditional on the allele frequency in the ancestral population (at the root) to be $k_{5} / K$, we obtain

$$
\begin{aligned}
L\left(\mathcal{D} ; \Theta \mid k_{5} / K\right)= & \left(\sum_{k_{3}=0}^{K} \mathbb{P}\left(X_{3} \in\left[l_{k_{3}}, u_{k_{3}}\right] \mid X_{5}=k_{5} / K,(t / 2 N)_{5 \rightarrow 3}\right) \mathbb{P}\left(z_{3} \mid n_{3}, k_{3}\right)\right) \\
& \cdot\left(\sum_{k_{4}=0}^{K} \mathbb{P}\left(X_{4} \in\left[l_{k_{4}}, u_{k_{4}}\right] \mid X_{5}=k_{5} / K,(t / 2 N)_{5 \rightarrow 4}\right)\right. \\
& \cdot\left(\sum_{k_{2}=0}^{K} \mathbb{P}\left(X_{2} \in\left[l_{k_{2}}, u_{k_{2}}\right] \mid X_{4}=k_{4} / K,(t / 2 N)_{4 \rightarrow 2}\right) \mathbb{P}\left(z_{2} \mid n_{2}, k_{2}\right)\right) \\
& \left.\cdot\left(\sum_{k_{1}=0}^{K} \mathbb{P}\left(X_{1} \in\left[l_{k_{1}}, u_{k_{1}}\right] \mid X_{4}=k_{4} / K,(t / 2 N)_{4 \rightarrow 1}\right) \mathbb{P}\left(z_{1} \mid n_{1}, k_{1}\right)\right)\right),
\end{aligned}
$$

and the full likelihood is obtained by summing over all possible ancestral frequencies

$$
L(\mathcal{D} ; \Theta, \pi)=\sum_{k_{5}=0}^{K} \pi\left(k_{5} / K\right) L\left(\mathcal{D} ; \Theta \mid k_{5} / K\right)
$$

The sums are slightly different when using beta with spikes, in order to correctly account for the loss and fixation probabilities.

Due to the binning, the above calculation provides an approximation which converges to the true likelihood as $K$ increases.

Polymorphic data The above likelihood calculation assumes that the data contains both sites that are polymorphic, and sites that are fixed or lost in all populations. However, SNP data is restricted to polymorphic sites. We can calculate the likelihood of the data 
conditional on observing only polymorphic sites as follows

$$
\begin{aligned}
L(\mathcal{D} ; \Theta, \pi \mid \text { polymorphism }) & =\frac{L(\mathcal{D} ; \Theta, \pi)}{\mathbb{P}(\text { polymorphism } \mid \Theta, \pi)}, \\
\mathbb{P}(\text { polymorphism } \mid \Theta, \pi) & =1-L\left(\mathcal{D}^{0} ; \Theta, \pi\right)-L\left(\mathcal{D}^{1} ; \Theta, \pi\right),
\end{aligned}
$$

where $\mathcal{D}^{0}$ and $\mathcal{D}^{1}$ are the data corresponding to the site being lost or fixed, respectively, in all samples from all populations

$$
\mathcal{D}^{0}=\left\{\left(0, n_{j}\right) \mid 1 \leq j \leq J\right\}, \quad \mathcal{D}^{1}=\left\{\left(n_{j}, n_{j}\right) \mid 1 \leq j \leq J\right\}
$$

The DAF at the root Let us assume that the DAF at the root is a beta with spikes distribution, with the sum of the spikes equal to $p_{\text {mono }}$ (i.e. the probability that the allele has either frequency 0 or 1$)$. Let $\pi$ denote the beta distribution over $(0,1)$. In the following, we show that under pure genetic drift, the likelihood conditional on polymorphic data is independent of $p_{\text {mono }}$.

We note that the probability of observing polymorphic data is zero if the allele frequency at the root is 0 or 1

$$
L(\mathcal{D} ; \Theta \mid 0)=L(\mathcal{D} ; \Theta \mid 1)=0
$$

from which

$$
L\left(\mathcal{D} ; \Theta, \pi, p_{\text {mono }}\right)=\left(1-p_{\text {mono }}\right) \sum_{k_{5}=1}^{K-1} \pi\left(k_{5} / K\right) L\left(\mathcal{D} ; \Theta \mid k_{5} / K\right),
$$

Similarly, for the unobserved monomorphic data we have that

$$
L\left(\mathcal{D}^{0} ; \Theta \mid 0\right)=L\left(\mathcal{D}^{1} ; \Theta \mid 1\right)=1, \quad L\left(\mathcal{D}^{0} ; \Theta \mid 1\right)=L\left(\mathcal{D}^{1} ; \Theta \mid 0\right)=0
$$


from which

$$
\begin{aligned}
& L\left(\mathcal{D}^{0} ; \Theta, \pi, p_{\text {mono }}\right)+L\left(\mathcal{D}^{1} ; \Theta, \pi, p_{\text {mono }}\right) \\
& \quad=p_{\text {mono }}+\left(1-p_{\text {mono }}\right)\left(\sum_{k_{5}=1}^{K-1} \pi\left(k_{5} / K\right) L\left(\mathcal{D}^{0} ; \Theta \mid k_{5} / K\right)+\sum_{k_{5}=1}^{K-1} \pi\left(k_{5} / K\right) L\left(\mathcal{D}^{1} ; \Theta \mid k_{5} / K\right)\right), \\
& \mathbb{P}\left(\text { polymorphism } \mid \Theta, \pi, p_{\text {mono }}\right) \\
& \quad=1-L\left(\mathcal{D}^{0} ; \Theta, \pi\right)-L\left(\mathcal{D}^{1} ; \Theta, \pi\right) \\
& \quad=\left(1-p_{\text {mono }}\right)\left(1-\sum_{k_{5}=1}^{K-1} \pi\left(k_{5} / K\right) L\left(\mathcal{D}^{0} ; \Theta \mid k_{5} / K\right)-\sum_{k_{5}=1}^{K-1} \pi\left(k_{5} / K\right) L\left(\mathcal{D}^{1} ; \Theta \mid k_{5} / K\right)\right) .
\end{aligned}
$$

Using the above, we obtain the likelihood under pure genetic drift conditional on polymorphism to be

$$
\begin{aligned}
L(\mathcal{D} ; \Theta, & \left.\pi, p_{\text {mono }} \mid \text { polymorphism }\right) \\
= & \frac{L\left(\mathcal{D} ; \Theta, \pi, p_{\text {mono }}\right)}{\mathrm{P}\left(\text { polymorphism } \mid \Theta, \pi, p_{\text {mono }}\right)} \\
= & \frac{\left(1-p_{\text {mono }}\right) \sum_{k_{5}=1}^{K-1} \pi\left(k_{5} / K\right) L\left(\mathcal{D} ; \Theta \mid k_{5} / K\right)}{\left(1-p_{\text {mono }}\right)\left(1-\sum_{k_{5}=1}^{K-1} \pi\left(k_{5} / K\right) L\left(\mathcal{D}^{0} ; \Theta \mid k_{5} / K\right)-\sum_{k_{5}=1}^{K-1} \pi\left(k_{5} / K\right) L\left(\mathcal{D}^{1} ; \Theta \mid k_{5} / K\right)\right)} \\
= & \frac{\sum_{k_{5}=1}^{K-1} \pi\left(k_{5} / K\right) L\left(\mathcal{D} ; \Theta \mid k_{5} / K\right)}{1-\sum_{k_{5}=1}^{K-1} \pi\left(k_{5} / K\right) L\left(\mathcal{D}^{0} ; \Theta \mid k_{5} / K\right)-\sum_{k_{5}=1}^{K-1} \pi\left(k_{5} / K\right) L\left(\mathcal{D}^{1} ; \Theta \mid k_{5} / K\right)} \\
= & L(\mathcal{D} ; \Theta, \pi \mid \text { polymorphism }) .
\end{aligned}
$$

We note that for all the simulations and inference results reported here and in the main text, we used only polymorphic data and the above conditional likelihood.

\section{Inference of divergence times: a simulation study}

Given a topology, we estimated the scaled branch lengths (under pure genetic drift) and the

DAF at the root by numerically maximizing the likelihood using the L-BFGS-B algorithm 
Table S1: Summary of normalized differences.

\begin{tabular}{llcccccc}
\hline & & min & 5 th per & median & mean & 95th per & max \\
\hline \multirow{2}{*}{ Scenario I } & Beta & 0.0002 & 0.0063 & 0.0537 & 0.0623 & 0.1453 & 0.1702 \\
& Beta with spikes & 0.0005 & 0.0027 & 0.0198 & 0.0257 & 0.0629 & 0.1096 \\
& Kim Tree & 0.0010 & 0.0037 & 0.0761 & 0.0910 & 0.2006 & 0.2254 \\
\hline \multirow{2}{*}{ Scenario II } & Beta & 0.0026 & 0.0241 & 0.1508 & 0.2947 & 0.8582 & 0.8753 \\
& Beta with spikes & 0.0015 & 0.0250 & 0.0922 & 0.1056 & 0.2536 & 0.4073 \\
& Kim Tree & 0.0015 & 0.0063 & 0.1184 & 0.2134 & 0.5735 & 0.6544 \\
\hline
\end{tabular}

The table shows the summary of the distribution of the absolute normalized difference $\left(\left|1-\tau_{\text {est }} / \tau\right|\right)$ between the inferred $\left(\tau_{\text {est }}\right)$ and true $(\tau)$ scaled branch lengths, for the two simulation scenarios and beta, beta with spikes and Kim Tree. For beta and beta with spikes, we used $T=30$ and $K=25$ and $K=20$ for scenarios I and II, respectively.

(Byrd et al. 1995) implemented in SciPy (Jones et al. 2001). For this, we treat the DAF at the root as a nuisance parameter assumed to be a beta distribution and estimated the two shape parameters.

To estimate the scaled branch lengths, we fixed the number of generations on each branch, estimated the population size and then reported the resulting scaled time. As presented in the parameter scaling section, if the population size is large enough, this approach should provide similar estimates independent of the chosen number of generations per branch.

For the tree depicted in Figure 2 in the main text, we set the total height (number of generations from the root to the present) to a given $T$ and the generations per branches to be $t_{5 \rightarrow 4}=t_{4 \rightarrow 1}=t_{4 \rightarrow 2}=T / 2$ and $t_{5 \rightarrow 3}=T$. We simulated data using two different scenarios (Table 1 in the main text).

A comparison between beta, beta with spikes and Kim Tree (Gautier and Vitalis 2013) is reported in the main text (Figure 3). Table S1 contains the summary of the quality of the estimates for all three methods for both simulation scenarios. Here, we discuss in more 
details the effect of the chosen height $T$ and number of bins $K$.

Figure S1 (A and B) illustrates the quality of the estimates from beta and beta with spikes, for different tree heights $T$ and number of bins $K$. One of the trends that is clear in the figure is that beta with spikes has a lower variance than beta in the estimated branches lengths. This is probably a result of the variability between the different simulated data sets of the number of sites that are close to being fixed or lost. This should have a stronger effect on the beta than the beta with spikes, as these sites require accurate probabilities close to the boundaries.

For scenario I, Figure S1 A indicates that using $K=25$ bins is enough to obtain a good approximation for the likelihood, as, for a fixed tree height $T$, the beta with spikes has similar performance for $K=25$ and larger values of $K$. The lower $K=10$ decreases the quality of the estimates just slightly for the beta with spikes, but the effect is more noticeable in the quality of the beta approximation. The different behavior of the beta and beta with spikes when comparing $K=10$ and $K=25$ might indicate that the likelihood approximation is more robust to the number of bins, provided that the boundary probabilities are treated separately (as in the case of the beta with spikes). For values of $K$ larger than 10, the beta distribution provides worse and worse estimates with an increased number of bins. This is most likely due to the more fine grained bins increasing the importance of accurately modeling the boundary probabilities, rendering worse results from the beta approximation.

We generally observe the same trends for both simulation scenarios (Figure S1 A and B), with the noticeable differences that: the average performance of beta and beta with spikes is lower for scenario II than scenario I; and the beta distribution has a surprisingly good performance for $K=5$ bins under scenario II. However, the likelihood of the inferred branches under the beta distribution with $K=5$ is approximately 30,000 units lower than the one under $K=20$, indicating a much lower support for the branches inferred using $K=5$.

The beta approximation provides just as good estimates regardless of the tree height $T$ 

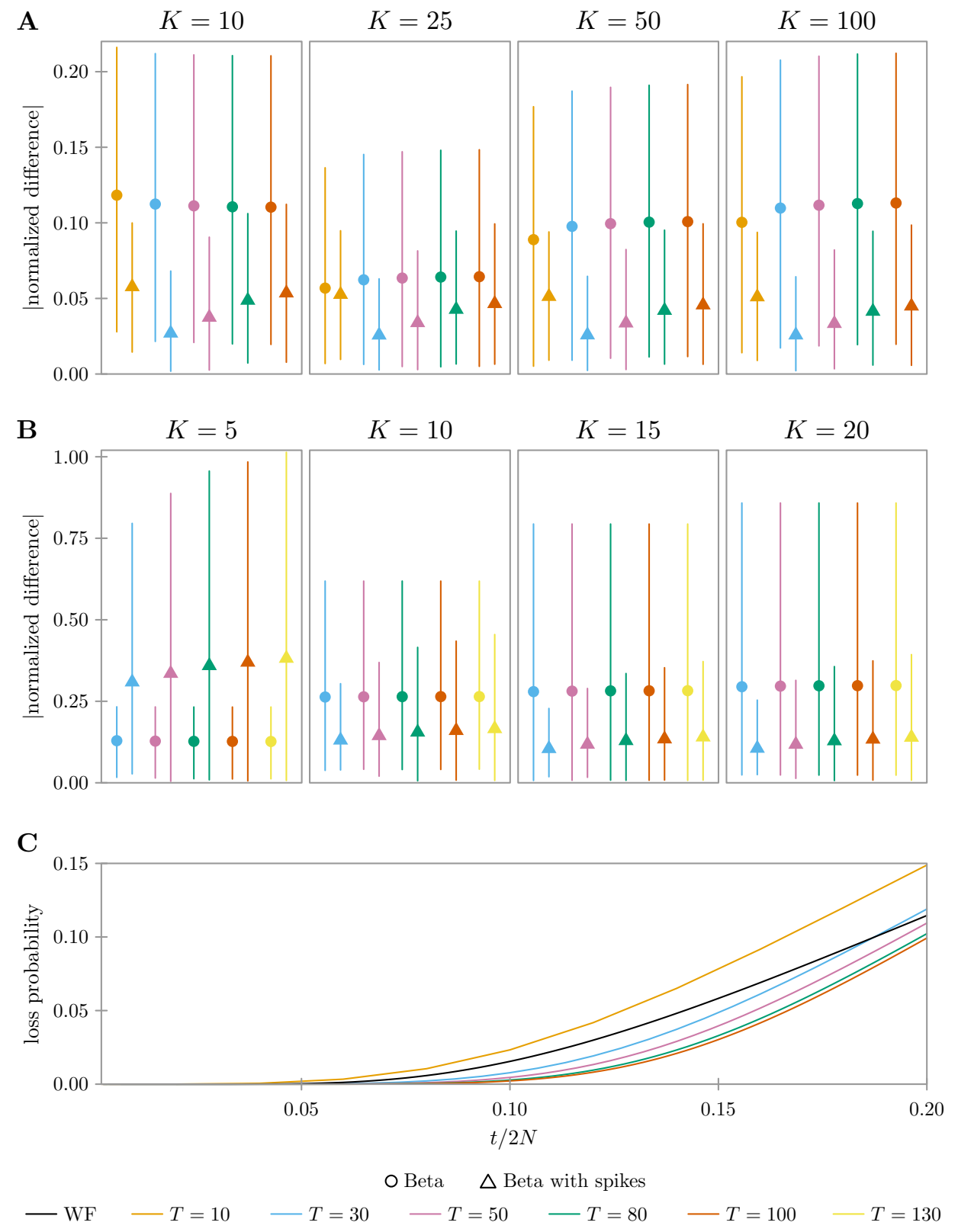

Figure S1: Effect of tree height $T$ and number of bins $K$. Absolute value of the normalized difference between the estimated branch length $\tau_{\text {est }}$ and the true $\tau$, given by $\left|1-\tau_{\text {est }} / \tau\right|$, for beta (circle) and beta with spikes (triangle), for scenarios I (A) and II (B). The plot indicates the mean over all 50 replicates for all four branch lengths, together with the 5th and 95th percentiles as error bars. (C) Loss probability as calculated from the Wright-Fisher (black) using $2 N=200$, initial frequency $x_{0}=0.2$ and generation times $t$ up to $t / 2 N=0.2$, and beta with spikes using different maximum generation times $T$ and corresponding population sizes $2 N$ such that $T / 2 N=0.2$. 
used, while the beta with spikes is more sensitive to the tree height. In this case, different tree heights would correspond to different scaling and $\Delta$, which is essentially a time step in a time discretization. The taller the tree, the more iterations are used in the recursion, allowing for more errors to accumulate from one iteration to the next. On the other hand, a tree that is too short leads to less accurate branch length inference. Here, a tree height of $T=30$ provided the best inference for both simulation scenarios, which contained trees with different true heights (Table 1 in main text), indicating that this height might be a good general choice regardless of the true underlying tree. Figure S1 C shows the effect of $T$ on the loss probability, illustrating that $T=30$ leads to the most accurate approximation of the loss probability.

For the results reported in Figure 3 and Table S1, we used $T=30, K=25$ and $K=20$ for scenarios I and II, respectively. As scenario II was built to generate chimpanzee-like data, we also used $T=30$ and $K=20$ for the results on the chimpanzee exome data reported in Figure 4 and Table 2.

We note here that the likelihoods reported in the main text in Table 2 were numerically maximized over the root DAF, while the branch lengths were kept constant.

\section{LITERATURE CITED}

Byrd, R. H., P. Lu, J. Nocedal, and C. Zhu, 1995 A limited memory algorithm for bound constrained optimization. SIAM Journal on Scientific Computing 16(5): 1190-1208.

Erdélyi, A., W. Magnus, F. Oberhettinger, and F. G. Tricomi, 1953 Higher transcendental functions, Volume 1. McGraw-Hill New York.

Ewens, W. J., 2004 Mathematical Population Genetics 1: I. Theoretical Introduction, Volume 27. Springer Science \& Business Media.

Felsenstein, J., 1981 Evolutionary trees from DNA sequences: a maximum likelihood approach. Journal of molecular evolution 17(6): 368-376. 
Gautier, M. and R. Vitalis, 2013 Inferring population histories using genome-wide allele frequency data. Molecular biology and evolution 30(3): 654-668.

Jones, E., T. Oliphant, P. Peterson, et al., 2001 SciPy: Open source scientific tools for Python. [Online; accessed 2014-04-03].

Sirén, J., 2012 Statistical models for inferring the structure and history of populations from genetic data. Ph. D. thesis, University of Helsinki, Faculty of Science, Department of Mathematics and Statistics. 